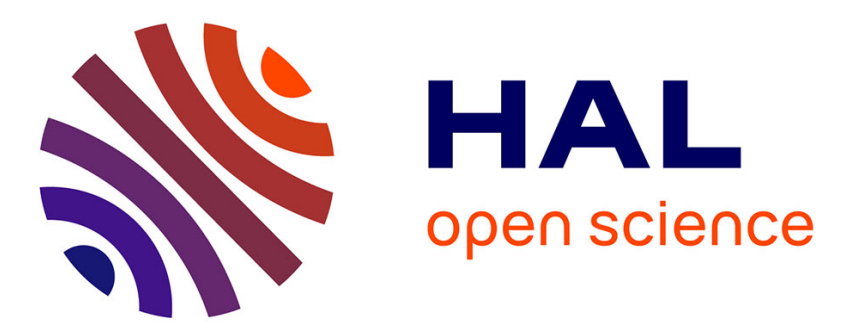

\title{
Spin excitations of two-dimensional-lattice electrons: Discussion of neutron-scattering and NMR experiments in high- $\mathrm{T}$ c superconductors
}

\author{
M. Lavagna, G. Stemmann
}

\section{- To cite this version:}

M. Lavagna, G. Stemmann. Spin excitations of two-dimensional-lattice electrons: Discussion of neutron-scattering and NMR experiments in high- T c superconductors. Physical Review B: Condensed Matter and Materials Physics (1998-2015), 1994, 49 (6), pp.4235 - 4250. 10.1103/PhysRevB.49.4235 . hal-01896252

\section{HAL Id: hal-01896252 \\ https://hal.science/hal-01896252}

Submitted on 16 Oct 2018

HAL is a multi-disciplinary open access archive for the deposit and dissemination of scientific research documents, whether they are published or not. The documents may come from teaching and research institutions in France or abroad, or from public or private research centers.
L'archive ouverte pluridisciplinaire HAL, est destinée au dépôt et à la diffusion de documents scientifiques de niveau recherche, publiés ou non, émanant des établissements d'enseignement et de recherche français ou étrangers, des laboratoires publics ou privés. 


\title{
Spin excitations of two-dimensional-lattice electrons: Discussion of neutron-scattering and NMR experiments in high- $T_{c}$ superconductors
}

\author{
M. Lavagna and G. Stemmann \\ Commissariat à l'Energie Atomique, Département de Recherche Fondamentale sur la Matiere Condensée, \\ SPSMS/MDN, 85X, 38041 Grenoble, France \\ (Received 26 July 1993)
}

\begin{abstract}
The spin-excitation spectrum as observed in neutron-diffraction and NMR experiments in $\mathrm{YBa}_{2} \mathrm{Cu}_{3} \mathrm{O}_{6+x}$ is theoretically examined in the weak-coupling limit of a two-dimensional Hubbard lattice model in which the next-nearest-neighbor hopping term $t^{\prime}$ is considered in order to fit the shape of the Fermi surface observed in angle-resolved-photoemission experiments. The presence of a Van Hove singularity in the density of states at $\omega=4 t^{\prime}$, no longer centered at the center of the band if $t^{\prime} \neq 0$, leads to correct predictions for the variation of the Knight shift with doping when compared to experiments. As long as pairing effects are not included, the frequency dependence of $\chi^{\prime \prime}(Q, \omega)$ at $T=0$ has no gap for the current regime of interest $\left(4 t^{\prime}<\mu<0\right)$, but instead presents a typical double-cusp structure that we discuss. In the presence of $d$-wave pairing, it is shown how the spectrum of excitations gains four additional superconducting ellipses around the nodes of excitations. Consequently at $T=0, \chi^{\prime \prime}(\mathbf{Q}, \omega)$ exhibits a resonance superimposed on a gap structure which bears striking resemblance with the neutron results reported by Rossat-Mignod et al. [Physica $\mathrm{B} \mathrm{169,} 58$ (1991)] in $\mathrm{YBa}_{2} \mathrm{Cu}_{3} \mathrm{O}_{6.92}$. The resonance is analyzed as a Kohn anomaly of the second kind in the Cooper channel. The value of the threshold $E_{G}$ evolves between two different regimes as the chemical potential goes from 0 to $4 t^{\prime}$, associated with two different scales for the temperature at which the spin gap fills up. Finally, the Knight shift is calculated showing a Yosida-like law with a linear $T$ dependence at very low temperatures as expected for axial superconductivity in two dimensions.
\end{abstract}

\section{INTRODUCTION}

Besides their high superconducting temperature, one of the most important observations made in the new superconductors concerns the aspect of the spin-excitation spectrum that they exhibit both above and below $T_{c}$. A very intensive effort has been made these past years to study the excitations in cuprates such as $\mathrm{La}_{2-x} \mathrm{Sr}_{x} \mathrm{CuO}_{4}$ (Ref. 1) or $\mathrm{YBa}_{2} \mathrm{Cu}_{3} \mathrm{O}_{6+x}$ (Refs. 2-5) using either neutron-scattering or NMR techniques. As far as neutron scattering is concerned, let us first summarize the essential results, well established for some of them or still under discussion for others.

(1) In the low-doping regime, both of these systems exhibit long-range antiferromagnetic order which disappears above a critical concentration of oxygen for yttrium compounds or, respectively strontium in lanthanum compounds. Nevertheless, in the metallic phase of yttrium compounds, the neutron-scattering signal keeps its maximum amplitude around the antiferromagnetic vector $\mathbf{Q}=(\pi, \pi)$ as is the case for antiferromagnetic commensurable fluctuations. The situation in lanthanum compounds is rather different with the onset of incommensurate peaks around the four wave vectors $\left(\pi, \pi \pm \delta q_{0}\right),\left(\pi \pm \delta q_{0}, \pi\right)$. In the following we will essentially discuss the case of yttrium compounds.

(2) The $q$ width of the neutron-scattering response around its maximum value is rather large, leading to a short magnetic correlation length $\xi$. Of particular importance is the fact that the correlation length does not ex- hibit any sizable variations with both temperature and frequency apart from the special behavior around the resonance outlined further below. On the other hand, one observes a continuous decrease of the magnetic correlation length $\xi$ when the concentration of oxygen increases. This last feature makes the measurements more and more difficult with increasing doping since the $q$ widening of the response is associated with a reduction of the signal itself at the wave vector $\mathbf{Q}$. In the following we will essentially discuss the frequency behavior of the magnetic form factor $S(\mathrm{Q}, \omega)$ in yttrium compounds.

(3) Well established now is the presence of a resonance in $S(\mathbf{Q}, \omega)$ for the superconducting phase of highly doped $\mathrm{YBa}_{2} \mathrm{Cu}_{3} \mathrm{O}_{6+x}$ systems at a characteristic frequency of the order of $41 \mathrm{meV}$. This resonance was first reported by Rossat-Mignod et al. ${ }^{2}$ for the concentrations $x=0.92$ and 1.0 and has recently been confirmed for the latter composition using polarized neutron-scattering techniques. ${ }^{5}$ The existence of this resonance has not received any interpretation so far, and it is one of the purposes of this paper to answer this question. The fact that the feature disappears above $T_{c}$ brings strong arguments in favor of a mechanism based on pairing effects. As we have already mentioned, this resonance is accompanied by a narrowing of the $q$ width, i.e., an increase of the magnetic correlation length in its vicinity.

(4) Still under discussion is the question concerning the existence of a gap in the spectrum of excitations $S(\mathbf{Q}, \omega)$ at low temperatures. The neutron-scattering measurements performed by the Grenoble group ${ }^{2}$ show a continuous behavior as a function of doping with the presence of 
a gap $E_{G}$ of values $4,16,28$, and $26 \mathrm{meV}$ at the concentrations $x=0.51,0.69,0.92$, and 1.0 , respectively. The temperature $T_{m}$ at which the gap structure disappears is experimentally identified as the temperature of the maximum of $\chi^{\prime \prime}\left(\mathbf{Q}, \omega_{0}\right)$ for $\omega_{0} \ll E_{G}$. This energy scale $T_{m}$ is found to be larger than $T_{c}$ for the first three compositions in question (and of order $130 \mathrm{~K}$ ), hence the appellation of spin gap (or pseudogap) in these systems. Oppositely, for surdoped systems $(x=1.0), T_{m}$ just equals $T_{c}$ (of order 90 $\mathrm{K})$ and the gap vanishes immediately above $T_{c}$ as the superconducting gap does in BCS theory. Note the apparently contradictory behavior of $E_{G}$ and $T_{m}$ with doping: $E_{G} / T_{c}$ increases with $x$, while $T_{m} / T_{c}$ decreases, reaching the critical value 1 for the upper $x=1.0$ concentration. Moreover, in the spin-gap regime, $E_{G}$ is rather small toward $2\left(4 \Delta_{0}\right)$, reaching a value of $3.5 T_{c}$ only in the surdoped regime. Because of the smallness of the signal itself (especially at higher doping for which the magnetic correlation length becomes shorter) and also from the difficulty of extracting the magnetic contribution, the controversy is still open on the existence of this spin gap. It has only been verified by other groups for the composition $x=0.6$ (Ref. 4) with the prediction of a gap of 5 $\mathrm{meV}$, properly interpolating between the values found by Rossat-Mignod et al. at the neighboring concentrations $x=0.51$ and 0.69 . All the other compositions have so far led to negative confirmations ${ }^{3,5}$ in spite of the natural arguments in favor of the existence of the spin gap provided by the low-temperature behavior of the relaxation time on ${ }^{63} \mathrm{Cu}$ nuclei as discussed below.

Of special interest in this discussion are the NMR experiments performed on the same systems. ${ }^{6,7}$ Depending on the nuclei considered $(\mathrm{Cu}, \mathrm{O}$, or $\mathrm{Y})$, the relaxation rates exhibit very different temperature dependence, reflecting the various $\mathbf{q}$ filterings involved in each case. For ${ }^{63} \mathrm{Cu}$, the relaxation time does not follow the usual Korringa law, but instead $\left(T_{1} T\right)^{-1}$ shows a maximum at $T_{m} \geq T_{c}$ in heavily doped systems. Again, this maximum coincides with the superconducting temperature only for surdoped systems $(x=1.0)$. Given that the form factor of ${ }^{63} \mathrm{Cu}$ mostly filters the $\mathrm{Q}$ component, it is currently accepted that the thermal dependence of the relaxation rate on copper is directly related to the formation of the spin gap in $S(Q, \omega)$. For ${ }^{17} \mathrm{O}$ (and also ${ }^{89} \mathrm{Y}$ ), a rather different behavior has been found with a regular decrease of ${ }^{17}\left(T_{1} T\right)^{-1}$ when lowering the temperature, starting from well above $T_{c}$ in heavily doped systems. For surdoped systems, ${ }^{17}\left(T_{1} T\right)^{-1}$ is larger and slightly increases in the normal phase when the temperature decreases before falling down just below $T_{c}$. The Knight shift is found to behave in a very similar way with a constant coefficient of proportionality toward ${ }^{17}\left(T_{1} T\right)^{-1}$.

The whole set of these experimental neutron-scattering and NMR data constitutes a puzzling problem in that the spin-excitation spectrum drastically differs from a traditional Fermi-liquid behavior. This triggered these last years the development of a certain number of theories. Apart from the phenomenological approaches, ${ }^{8,9}$ let us quote the nested-Fermi-liquid (NFL) theories which have been derived either in the weak-coupling regime $\mathrm{e}^{10-12}$ or in the strong-coupling regime $e^{13,14}$ of various magnetic models (Hubbard, $t-J$ ) appropriate to describe the electronic configuration of cuprate layers. All these theories invoke a dynamic nesting property due to the existence of flat parts of the Fermi surface. They predict the existence of a gap in the frequency dependence of $\chi_{0}^{\prime \prime}(\mathbf{Q}, \omega)$ of value $2|\mu|$, followed by all the larger jumps since the system is closer to the Van Hove singularity of the density of states existing at half-filling. The NFL theories especially in their strong-coupling version lead to interesting results concerning the existence of a spin gap in $\chi_{0}^{\prime \prime}(\mathbf{Q}, \omega)$, the nonmonotonic behavior of ${ }^{63}\left(T_{1} T\right)^{-1}$ with temperature, and the prediction of short magnetic correlation lengths only weakly dependent on temperature. However, they do not succeed in giving the correct order of evolution of the Knight shift with doping. Even more seriously, recent results of photoemission experiments ${ }^{15}$ have drastically challenged these models by bringing definitive proof on the existence of the Fermi surface of shape in complete contradiction with the starting hypothesis of NFL theories. Precisely, the Fermi surface is found to be rotated an angle of $45^{\circ}$ toward the assumed picture and its center moved from the points $\Gamma(0,0)$ to $S(\pi, \pi)$ of the Brillouin zone.

Whatever will be the final issue concerning the experimental controversy about the existence of the spin gap, which is, of course, crucial to settling in the future for a better understanding of the high- $T_{c}$ superconductors, it appeared urgent on the theoretical side to reexamine the question without any a priori assumptions on the existence of the spin gap and by taking into account the full realistic shape of the Fermi surface as emerged from photoemission experiments and confirmed by band-structure calculations. This constitutes the purpose of this paper with regards to the weak-coupling regime which has the interest of already bringing out the main features of the problem. The strong-coupling limit will be considered in a forthcoming paper. ${ }^{18}$ The first part of this paper is devoted to the study of the spin excitations in the normal phase of a $t-t^{\prime}$ Hubbard model in which the next-nearest hopping term $t^{\prime}$ is introduced to reproduce the correct shape of the Fermi surface. The Van Hove singularity already present in the $t^{\prime}=0$ case is then pushed from the center of the band to $\omega=4 t^{\prime}$, which makes the model a much better starting point from a perturbative point of view. Results for the spin-excitation spectrum are established with special emphasis on what becomes of the dynamic nesting property and of the spin gap at $Q=(\pi, \pi)$. In the second part, the discussion is enlarged to the presence of $d$-wave superconductivity. We show how the formation of four superconducting ellipses in the spectrum of excitations deeply affects the form of the dynamic susceptibility at low frequencies with the prediction of a gap followed by a resonance which bears striking resemblance to the neutron-scattering results mentioned before. Moreover, the combined effects of the anisotropy of the superconducting gap as well as that of the Fermi surface have consequences on the establishment of very different regimes for the dynamic susceptibility as the chemical potential goes from 0 to $4 t^{\prime}$, leading to a typical variation of $E_{G}$ and $T_{m}$ with doping, which might be of some relevance to account for the experiments. 


\section{NORMAL STATE}

Let us start from the effective one-hand Hubbard Hamiltonian ${ }^{16,17}$ considered to describe the $\mathrm{Cu}-\mathrm{O}$ singlets contained in the layers:

$H=-\sum_{\langle i, j\rangle, \sigma} t f_{i \sigma}^{\dagger} f_{j \sigma}-\sum_{\langle i, j\rangle^{\prime}, \sigma} t^{\prime} f_{i \sigma}^{\dagger} f_{j \sigma}+U \sum_{i} n_{i \uparrow} n_{i \downarrow}$,

where $t$ and $t^{\prime}$ are the hopping integrals between nearest neighbors $\langle i, j\rangle$ and next-nearest neighbors $\langle i, j\rangle^{\prime}$. The

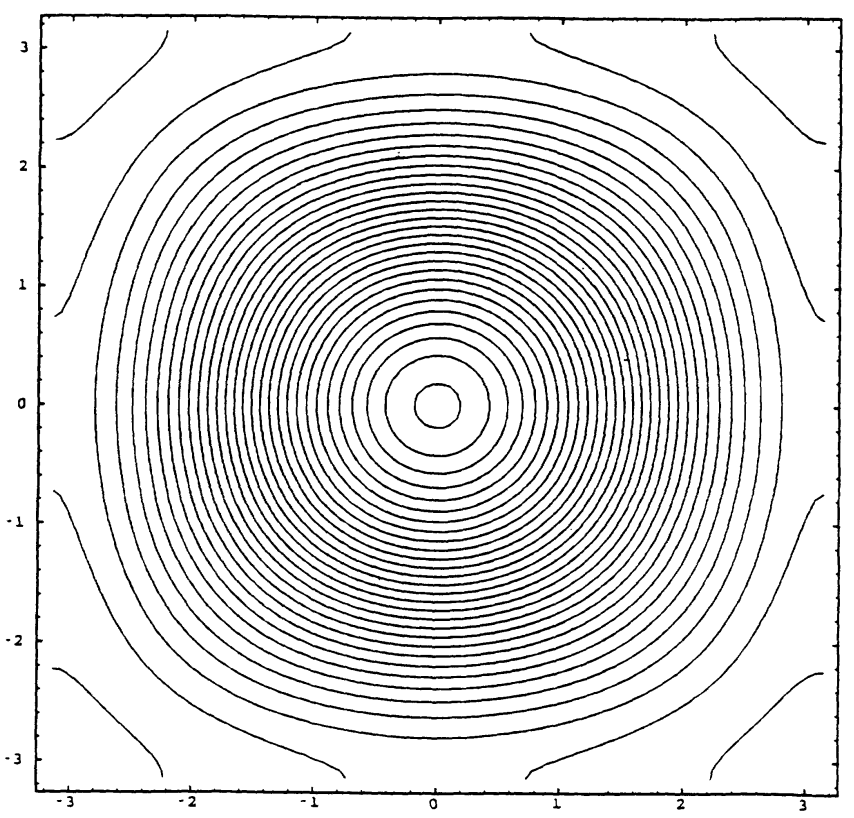

(a)

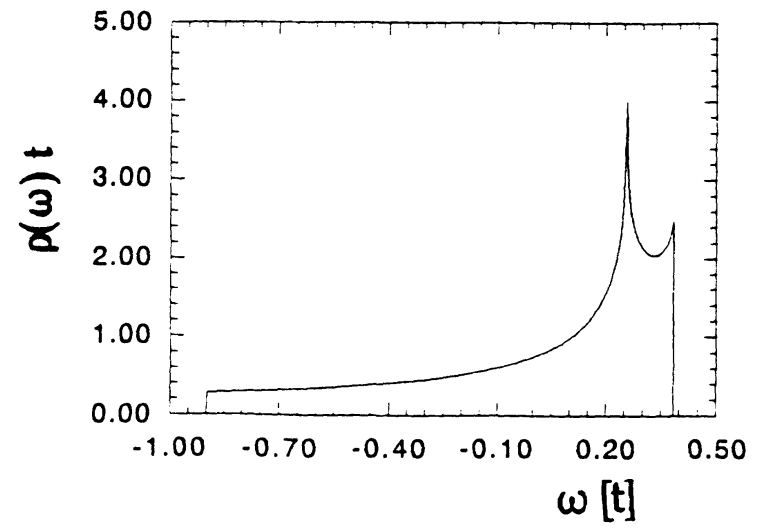

(b)

FIG. 1. Tight-binding model with positive next-nearestneighbor hopping integral $t^{\prime}$. (a) Energy contours for $t^{\prime}=+0.45 t$. Note the open orbits for $\varepsilon>4 t^{\prime}$ and the closed orbits for $\varepsilon<4 t^{\prime}$ rotated by $45^{\circ}$ compared to the case $t^{\prime}=0$ and centered around $(0,0)$. (b) Density of states for the same $t^{\prime}$ and $t=0.16$. The Van Hove singularity is located at $\omega=4 t^{\prime}$. Note also that the case of half filling corresponds to a positive value of $\mu$ smaller than $4 t^{\prime}$ so that the density of states decreases with hole doping. latter one is introduced to fit the shape of the Fermi surface $\Sigma_{F}$ observed in angle-resolved-photoemission experiments. These results ${ }^{15}$ confirmed by band-structure calculations show a rotation of the axes of symmetry of $\Sigma_{F}$ of an angle of $45^{\circ}$ compared to the $t^{\prime}=0$ case, with its center moved from the point $\Gamma(0,0)$ to $S(\pi, \pi)$. $U$ represents the on-site repulsion between particles. Typically, $t$ is of order of $0.5 \mathrm{eV},\left|t^{\prime}\right|$ varies between $0.1 t$ and $0.4 t$ (we will see further that only negative values are relevant), and $U$ is of order $5 \mathrm{eV}$.

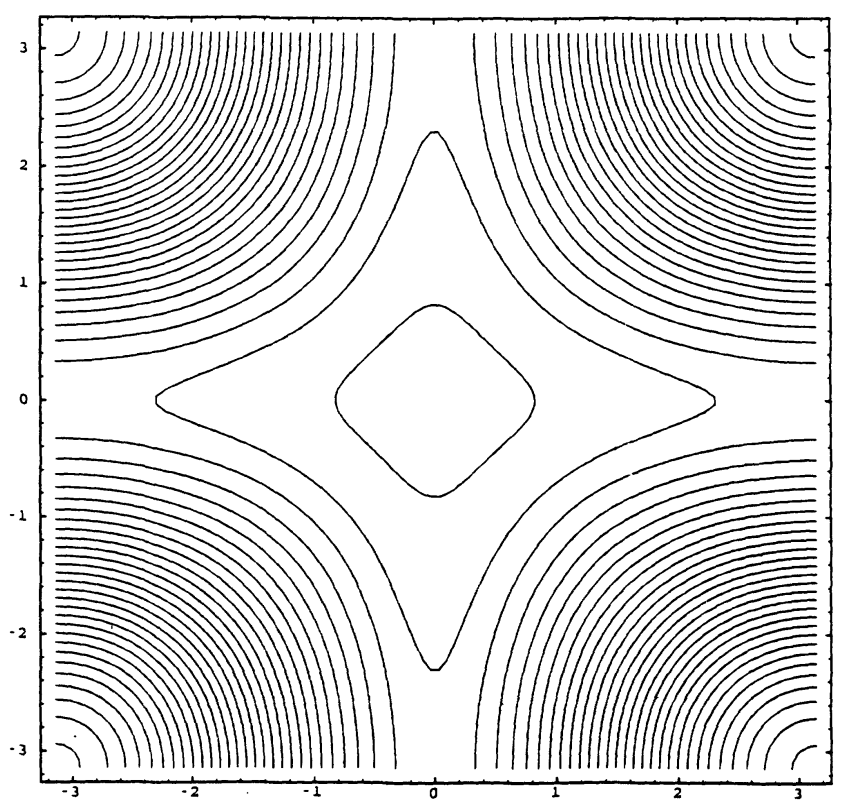

(a)

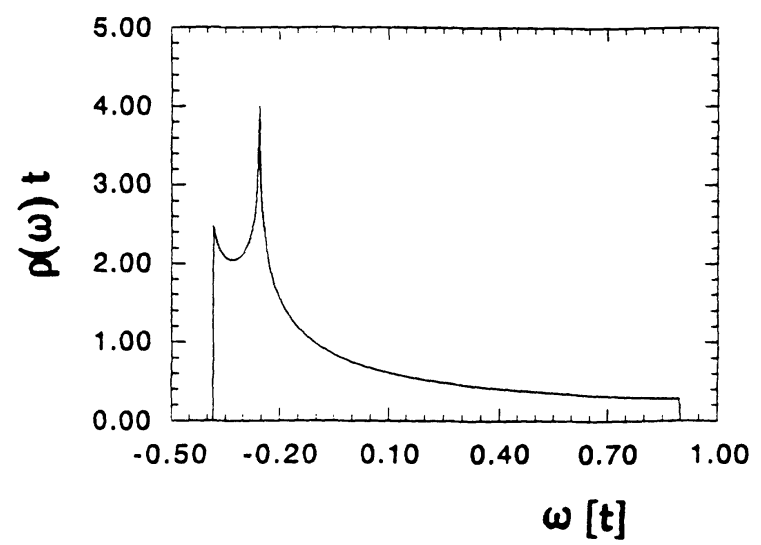

(b)

FIG. 2. Tight-binding model with negative next-nearestneighbor hopping integral $t^{\prime}$. (a) Energy contours for $t^{\prime}=-0.45 t$. Note that the closed orbits $\left(\varepsilon<4 t^{\prime}\right)$ are still centered around $(0,0)$ and no longer rotated, but the open orbits $\left(\varepsilon>4 t^{\prime}\right)$ are centered around $(\pi, \pi)$ and rotated by $45^{\circ}$. (b) Density of states for the same $t^{\prime}$ and $t=0.16$. The Van Hove singularity is located at $\omega=4 t^{\prime}$. In this case half filling corresponds to a negative value of $\mu$ greater than $4 t^{\prime}$ so that the density of states increases with hole doping. 
For a square-lattice structure, the spectrum of individual excitations in the normal state in the absence of any interaction effects is given by

$$
\xi_{k}=-2 t\left(\cos k_{x}+\cos k_{y}\right)-4 t^{\prime} \cos k_{x} \cos k_{y} .
$$

The corresponding equienergetics are represented in Figs. 1 and 2 . The spectrum is radically different depending on the sign of $t^{\prime}$. Obviously, the $t^{\prime}<0$ regime offers the better description of yttrium compounds and will be retained further. We have performed numerical calculations of the density of states and reported the results in Figs. 1 and 2 for both positive and negative values of $t^{\prime}$. Note the values of the band edges $\left(-4 t-4 t^{\prime}\right)$ and $\left(+4 t-4 t^{\prime}\right)$ and the presence of a Van Hove singularity at $\omega=4 t^{\prime}$ (different from zero) with an asymmetry compared to the $t^{\prime}=0$ case since the singularity is no longer associated with half-filling and makes the model much less pathological. The corresponding $\omega=4 t^{\prime}$ equienergetics is the "star" appearing in Fig. 2(a), the ends of which are the saddle points located at $( \pm \pi, 0)$ and $(0, \pm \pi)$. Below or above this critical value $\omega=4 t^{\prime}$, the equienergetics are, respectively, closed orbits centered around the point $\Gamma(0,0)$ or open orbital around $S(\pi, \pi)$.

At the unrenormalized level, the imaginary part of the unrenormalized dynamic susceptibility is given by

$\chi_{0}^{\prime \prime}(\mathbf{Q}, \omega)=-\pi \sum_{k}\left[n_{F}\left(\xi_{k}\right)-n_{F}\left(\xi_{k+Q}\right)\right] \delta\left(\omega-\xi_{k+Q}+\xi_{k}\right)$.

Results of our numerical calculations are reported in Figs. 3 and 4, respectively, at zero end finite temperatures. Let us remark that the presence of the $t^{\prime}$ term deeply affects the frequency dependence of $\chi_{0}^{\prime \prime}(\mathbf{Q}, \omega)$. The

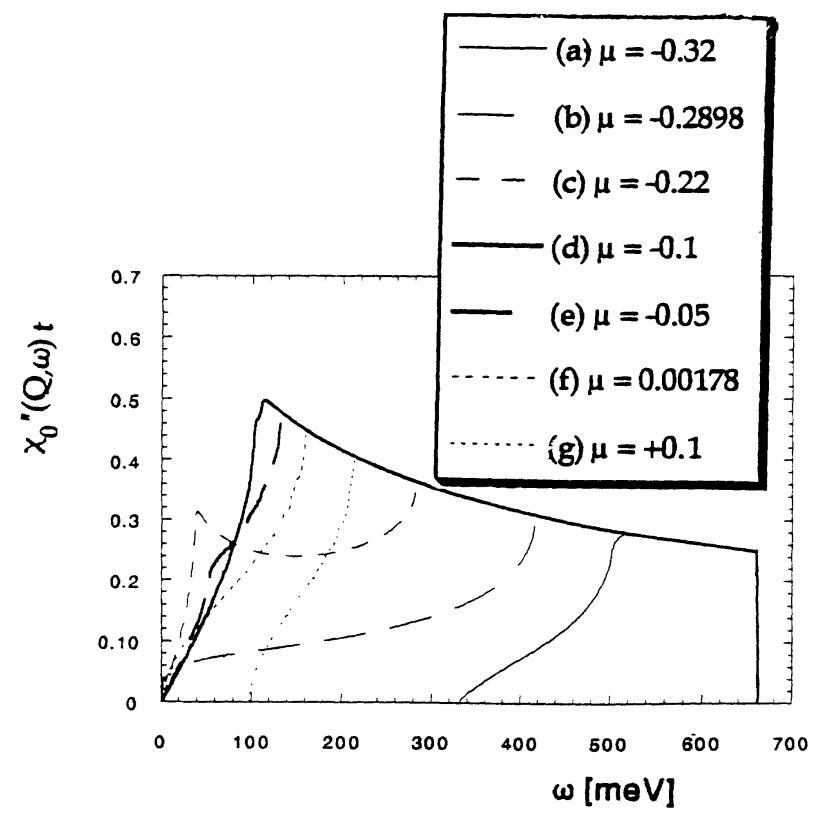

FIG. 3. Spectral weight $\chi_{0}^{\prime \prime}(\mathbf{Q}, \omega)$ vs frequency $\omega$ without pairing at zero temperature: $t=75 \mathrm{meV}, t^{\prime}=-0.45 t$. The enumeration of curves is in accordance with Table I.

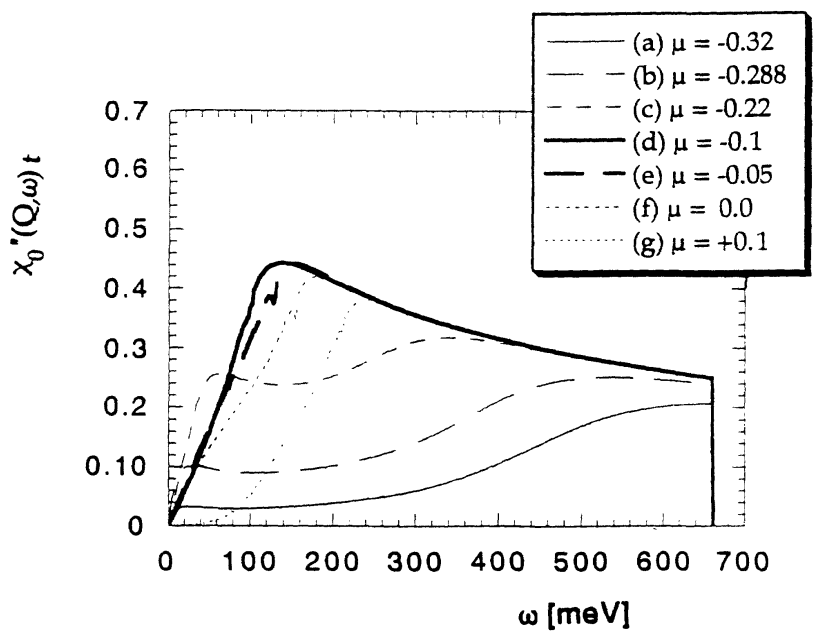

FIG. 4. Spectral weight $\chi_{0}^{\prime \prime}(\mathbf{Q}, \omega)$ vs frequency $\omega$ without pairing at $T=300 \mathrm{~K}, t=75 \mathrm{meV}, t^{\prime}=-0.45 t$. The enumeration of curves is the same as in Fig. 3.

dynamic nesting property observed at $t^{\prime}=0$ with the existence of a gap of value $2|\mu|$ is now replaced by the following behavior: (i) The two opposite regimes (closed orbits at $\mu<4 t^{\prime}$ or "small open orbits" at $\mu>0$ ) lead to the formation of a gap in the frequency dependence of $\chi_{0}^{\prime \prime}(\mathbf{Q}, \omega)$. (ii) "Large open orbits" such as $4 t^{\prime}<\mu<0$ show no gap but instead a characteristic "double-cusp" structure as shown in Fig. 3.

Our results at zero temperature are in agreement with the analytical results derived by Bénard, Chen, and Tremblay in a recent report. ${ }^{16}$ However, while the method they used is restricted to zero temperature, the numerical approach that we developed has no limitation of this type and has been as well applied to finite temper-

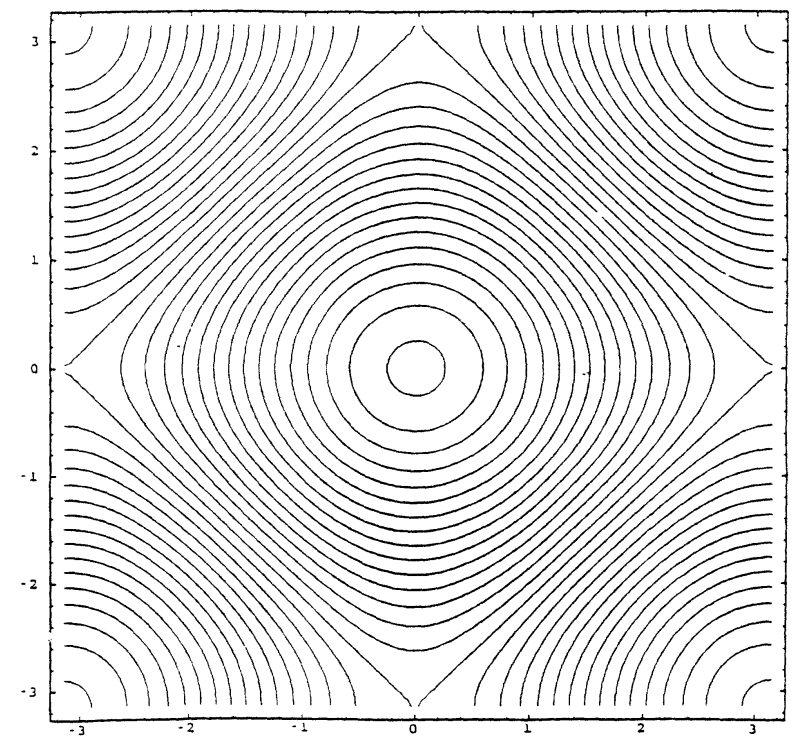

FIG. 5. Equienergetics $\xi_{k+Q}-\xi_{k}=$ const $[\mathbf{Q}=(\pi, \pi)]$ corresponding to spin-excitation energies in the normal phase whatever $t^{\prime}$ is. 
atures as shown in Fig. 4. We propose here a simple geometrical interpretation of the results. The density of excitations of electron-hole pairs of momentum transfer $Q$ derived from $\xi_{k+Q}-\xi_{k}=4 t\left(\cos k_{x}+\cos k_{x}\right)$ is not affected by the presence of the $t^{\prime}$ term and its corresponding spectrum is represented in Fig. 5. The density of pair excitations can then be expressed in terms of complete elliptic integrals with a characteristic Van Hove singularity at $\omega=0$. In order to get to $\chi_{0}^{\prime \prime}(\mathbf{Q}, \omega)$, one has to consider the additional effect of the Fermi factor $\left[n_{F}\left(\xi_{k}\right)-n_{F}\left(\xi_{k}+Q\right)\right]$ which acts as an extinction factor at zero temperature.

For $t^{\prime}=0$, the boundary of the extinction factor exactly coincides with the equienergetics $\xi_{k+Q}-\xi_{k}=2|\mu|$ (cf. Fig. 6) and the result of dynamic nesting derived by Bulut and Scalapino ${ }^{10}$ and developed further ${ }^{12,14,16}$ can be easily deduced from the figure (for $\omega>0$ ):

$$
\chi_{0}^{\prime \prime}(\mathbf{Q}, \omega)=\frac{\pi}{2} \theta(\omega-2|\mu|) \rho_{0}(\omega / 2),
$$

with

$$
\rho_{0}(\omega)=\frac{1}{2 \Pi^{2} t} K\left[\sqrt{1-\left(\omega / 4 t^{\prime}\right)^{2}}\right]
$$

where $K(x)$ is the complete elliptic integral of the first kind. In this case a threshold in the spectrum of excitations $\chi_{0}^{\prime}(\mathbf{Q}, \omega)$ of value $2|\mu|$ followed by all the larger jumps arises since the system is close to the Van Hove singularity in the density of states existing at half-filling.

The $t^{\prime} \neq 0$ case differs by the shape of the Fermi factor. One can distinguish three regimes as represented in Fig. 7.

(i) For $\mu<4 t^{\prime}$ (closed orbits), the delimitations of the extinction Fermi factors are given by the curves $\Gamma\left(\xi_{k}=\mu\right)$ and $\Gamma^{\prime}\left(\xi_{k+Q}=\mu\right)$ shown in Fig. 7(a). For $\omega>\omega_{0}\left(\omega_{0}\right.$ corresponding to the equienergetics passing through points of type $A$ ), the Fermi factor does not have any extinction role and the response is the full complete elliptic integral as in the case for $t^{\prime}=0$. For $\omega_{c}^{+}<\omega<\omega_{0}\left(\omega_{c}^{+}\right.$for points of type $\left.B\right)$, part of the complete elliptic integral is made extinct, leading to an incomplete elliptic integral form for the response and a cusp in the frequency dependence of $\chi_{0}^{\prime \prime}(\mathbf{Q}, \omega)$. For $\omega<\omega_{c}^{+}$, we have $\chi_{0}^{\prime \prime}(\mathbf{Q}, \omega)=0$. Simple geometric arguments give the values of the threshold $\omega_{c}^{+}$and of the position of the cusp at $\omega_{0}$ :

$$
\omega_{c}^{+}=2\left[\frac{4 t^{\prime}-\mu}{1+\frac{2 t^{\prime}}{t}}\right],
$$
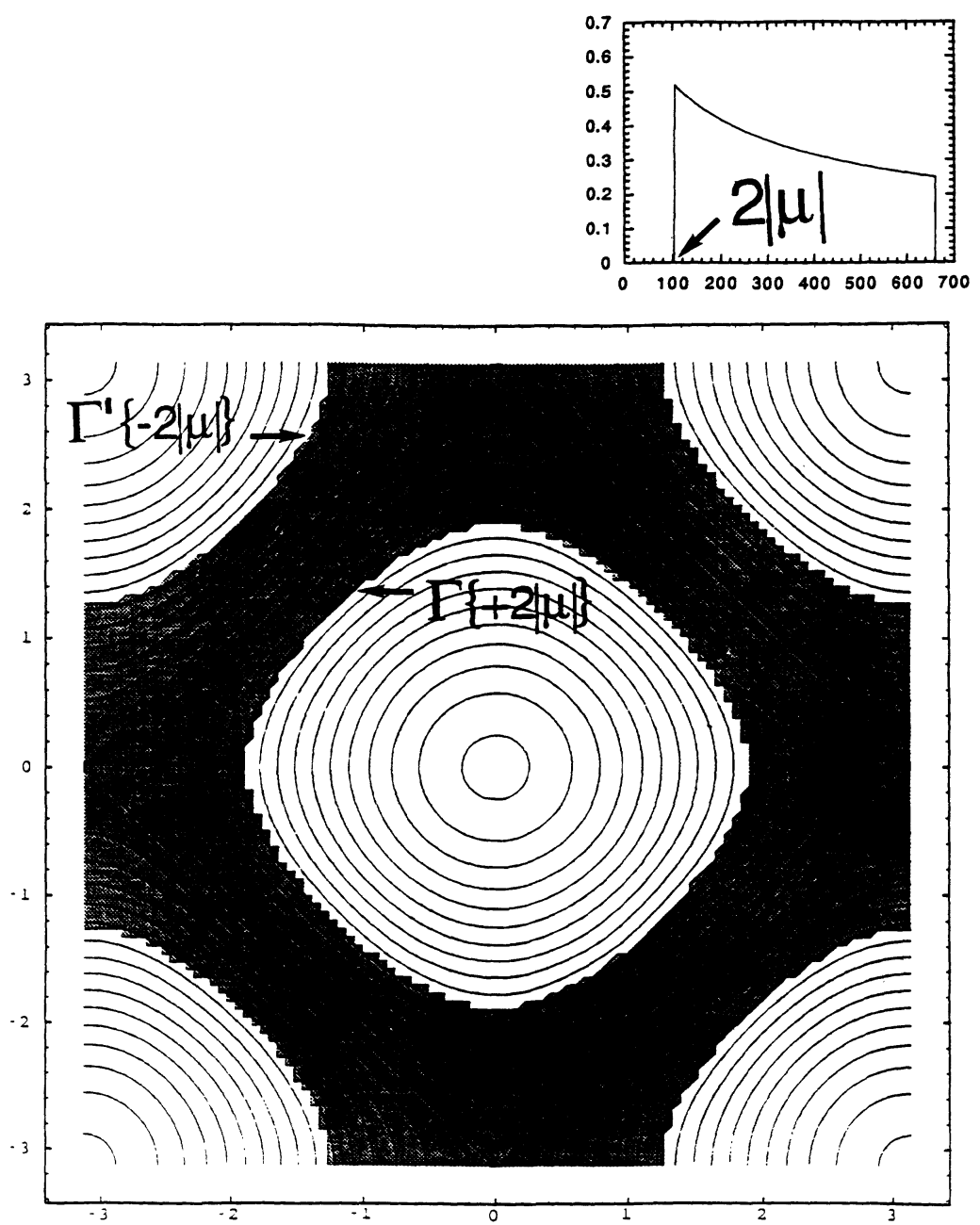

FIG. 6. Equienergetics $\xi_{k+Q}-\xi_{k}=$ const without pairing. The shaded region does not contribute to $\chi_{0}^{\prime \prime}(\mathbf{Q}, \omega)$ because of the extinction role played by the Fermi factor between the curves $\Gamma$ and $\Gamma^{\prime}$. In the inset, spectral weight $\chi_{0}^{\prime \prime}(\mathbf{Q}, \omega)$ vs frequency $\omega$ without pairing at $T=0$ for $t^{\prime}=0$. For $\mu \neq 0$ the vector $\mathbf{Q}$ no longer spans the Fermi surface and a gap of value $2|\mu|$ opens up. 


$$
\omega_{0}=\frac{4 t^{2}}{-t^{\prime}}\left[1-\left(1-\frac{\mu t^{\prime}}{t^{2}}\right)^{1 / 2}\right] .
$$

These values already play a role in the analytic expressions obtained in Ref. 16 for $T=0$.

(ii) For $4 t^{\prime}<\mu<0$ (large open orbits), the discussion is rather different as illustrated in Fig. 7(b). Contributions of points of type $C$ associated with $\omega=0$ are responsible for a finite response as soon as $\omega \neq 0$ and there is no gap. Instead, we find a double-cusp structure at the values $\omega_{0}$ and $\omega_{c}^{-}$(corresponding to points of types $D$ and $E$ ) defined by

$$
\omega_{c}^{-}=2\left[\frac{\mu-4 t^{\prime}}{1-\frac{2 t^{\prime}}{t}}\right] .
$$

Note that there is a critical value of $\mu$ for which the two values $\omega_{0}$ and $\omega_{c}^{-}$pass each other.

(iii) For $\mu>0$ (small open orbits), we find again a threshold in the spectrum of excitations of value $\Omega_{0}$ [associated with points of type $F$ of Fig. 7(c)] followed by a cusp at $\omega_{c}^{-}$(point $G$ ), beyond which a complete elliptic integral form is recovered for $\chi_{0}^{\prime \prime}(\mathbf{Q}, \omega)$. We have

$$
\Omega_{0}=\frac{4 t^{2}}{\left(-t^{\prime}\right)}\left[\left(1-\frac{\mu t^{\prime}}{t^{2}}\right]^{1 / 2}-1\right] \text {. }
$$

Table I summarizes the discussion of the gap and cusps in $\chi_{0}^{\prime \prime}(\mathbf{Q}, \omega)$ in the different regimes. The values of $\omega_{0}, \omega_{c}^{-}$, $\omega_{c}^{+}$, and $\Omega_{0}$ are in perfect quantitative agreement with our numerical calculations. The existence of a gap at zero temperature in the two opposite regimes $\left(\mu<4 t^{\prime}\right.$ and $\mu>0$ ) is simply due to the fact that the vector $\mathbf{Q}$ is, respectively, larger or smaller than any diagonal vector $\left(q_{x}=q_{y}\right)$ spanning the Fermi surface. For the intermediate regime $4 t^{\prime}<\mu<0$, which is effectively the case for the range of doping of physical systems, the threshold vanishes and instead a double-cusp structure takes place. The effect of finite temperatures is to fill up the gap when it exists and smooth the cusp structure as indicated in Fig. 4, pushing the second cusp to higher values.

The origin of the large values reached by $\chi_{0}^{\prime \prime}(\mathbf{Q}, \omega)$ is closely related to the Kohn anomalies of the second kind observed in $\chi_{0}^{\prime}(q, \omega=0)$ at $q=2 k_{F}$. Typically, one can write by changing the variables $\left(\xi_{k}\right.$ and $\left.\xi_{k+q}\right)$ :
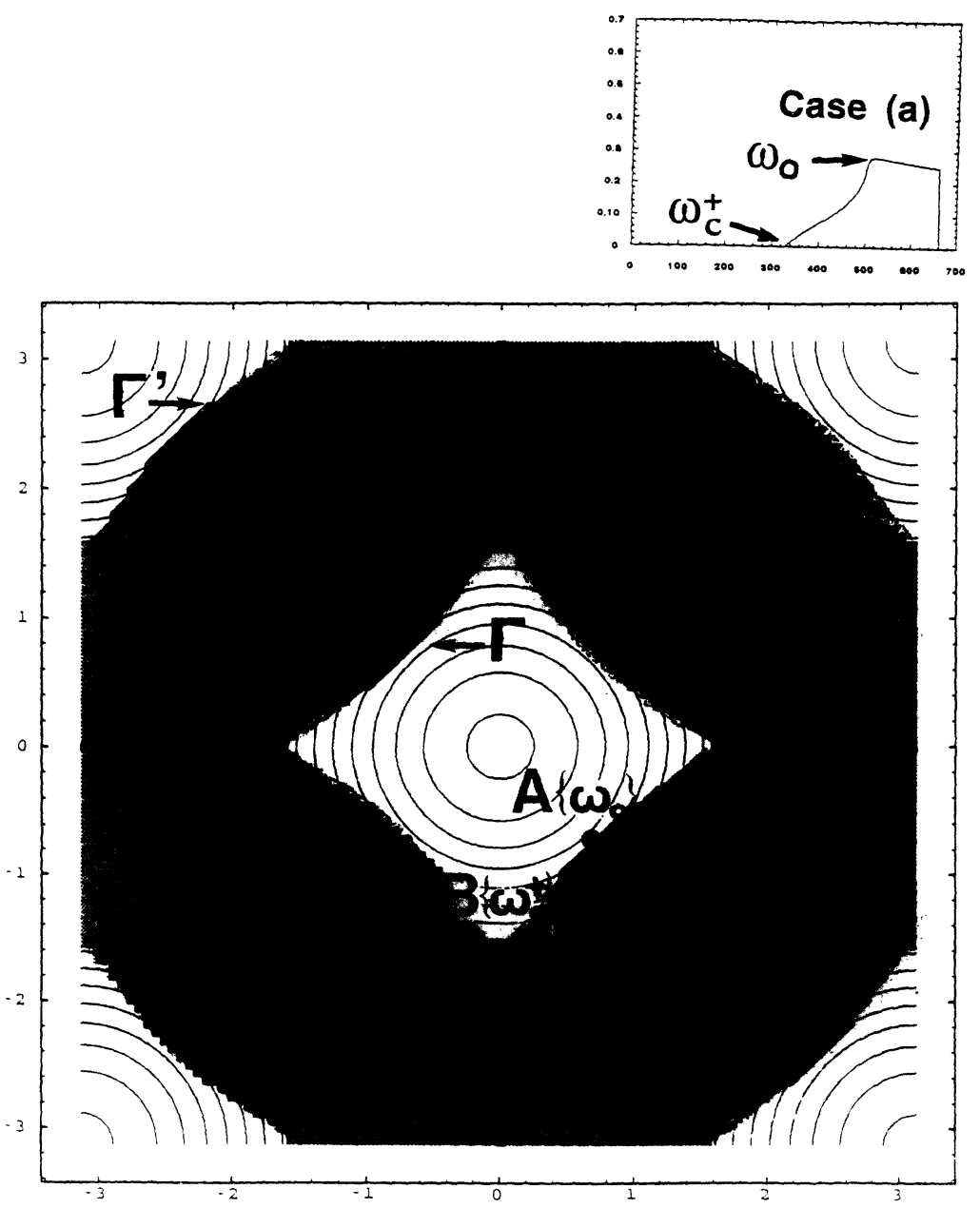

(a)
FIG. 7. Equienergetics $\xi_{k+Q}-\xi_{k}=$ const without pairing at $T=0$ for $t=75 \mathrm{meV}$, $t^{\prime}=-0.45 t$. (a) case (a) $\mu<4 t^{\prime}$ (closed orbits). (b) case (c) $4 t^{\prime}<\mu<\mu_{c 1}$ (large open orbits). (c) case (g) $\mu>0$ (small open orbits). The convention used for the shaded regions is the same as in Fig. 6. In the inset, spectral weight $\chi_{0}^{\prime \prime}(Q, \omega)$ vs frequency in each case. 


$$
\begin{aligned}
\chi_{0}^{\prime}(\mathbf{q}, \omega=0)=\iint d k_{x} d k_{y} \frac{n_{F}\left(\xi_{k+q}\right)-n_{F}\left(\xi_{k}\right)}{\xi_{k+q}-\xi_{k}} \\
=\iint \frac{d \xi_{k+q} d \xi_{k}}{\left|\nabla_{k} \xi_{k+Q} \wedge \nabla_{k} \xi_{k}\right|} \\
\times \frac{n_{F}\left(\xi_{k+q}\right)-n_{F}\left(\xi_{k}\right)}{\xi_{k+q}-\xi_{k}} .
\end{aligned}
$$

The Kohn anomalies arise when the vector $\mathbf{q}$ connects points of the Fermi surface for which tangents are parallel, leading to a zero value of the Jacobian present in the denominator of the expression above and, hence, a singularity in $\chi_{0}^{\prime}(\mathbf{q}, \omega=0)$. The Kohn anomalies are of the first kind for spherical symmetry. They are more pronounced and become of the second kind when the same property stands not only for points but for lines of the Fermi surface. This is the case for the anisotropic Fermi surface which has quasiflat parts in parallel (nesting property). This property for $\chi_{0}^{\prime}(\mathbf{q}, \omega=0)$ also reflects on the $\mathbf{q}$ dependence of $\chi_{0}^{\prime \prime}(\mathbf{q}, \omega \approx 0)$ : An equivalent way to look at it is to relate this property to the proximity of a Van Hove singularity for the density of excitations of electron-hole pairs arising from $\delta\left(\xi_{k+q}-\xi_{k}\right)$. The dynamical nesting property observed in $\chi_{0}^{\prime \prime}(\mathbf{Q}, \omega)$ is nothing but the dynamic version of the static Kohn anomalies generalized to finite frequencies through $\delta\left(\omega-\xi_{k+q}+\xi_{k}\right)$. It exists in both situations considered here $\left(t^{\prime}=0\right.$ or not), while the existence of a gap in the spectrum of excitations depends on the exact details of the structure. The gap always exists for $t^{\prime}=0$, while for $t^{\prime} \neq 0$ the gap structure is lost in the intermediate regime $4 t^{\prime}<\mu<0$, which is likely realized in the physical systems $\mathrm{YBa}_{2} \mathrm{Cu}_{3} \mathrm{O}_{6+x}$ for the range of concentration considered and for reasonable choices of $t^{\prime} / t$. This is contradicted by some of the neutron-scattering experiments ${ }^{2,5}$ which show the existence of a spin gap in a large range of concentration from $x=0.52$ to 1.0. An alternative way to reconcile the information coming from photoemission (shape of the Fermi surface compatible with a given value of the ratio $\left.t^{\prime} / t\right)$ and those given by neutron-scattering experiments (existence of a spin gap) would be to consider the additional effect of spinon pairing in the spirit of the so-called resonant valence bond (RVB) advanced by some authors ${ }^{19}$ in the earlier years of high- $T_{c}$ superconductors. We will postpone this discussion to our forthcoming paper ${ }^{18}$ where we offer to study the effect of spinon pairing in the normal phase of a $t-t^{\prime}-J$ model which constitutes in one sense the strong-coupling
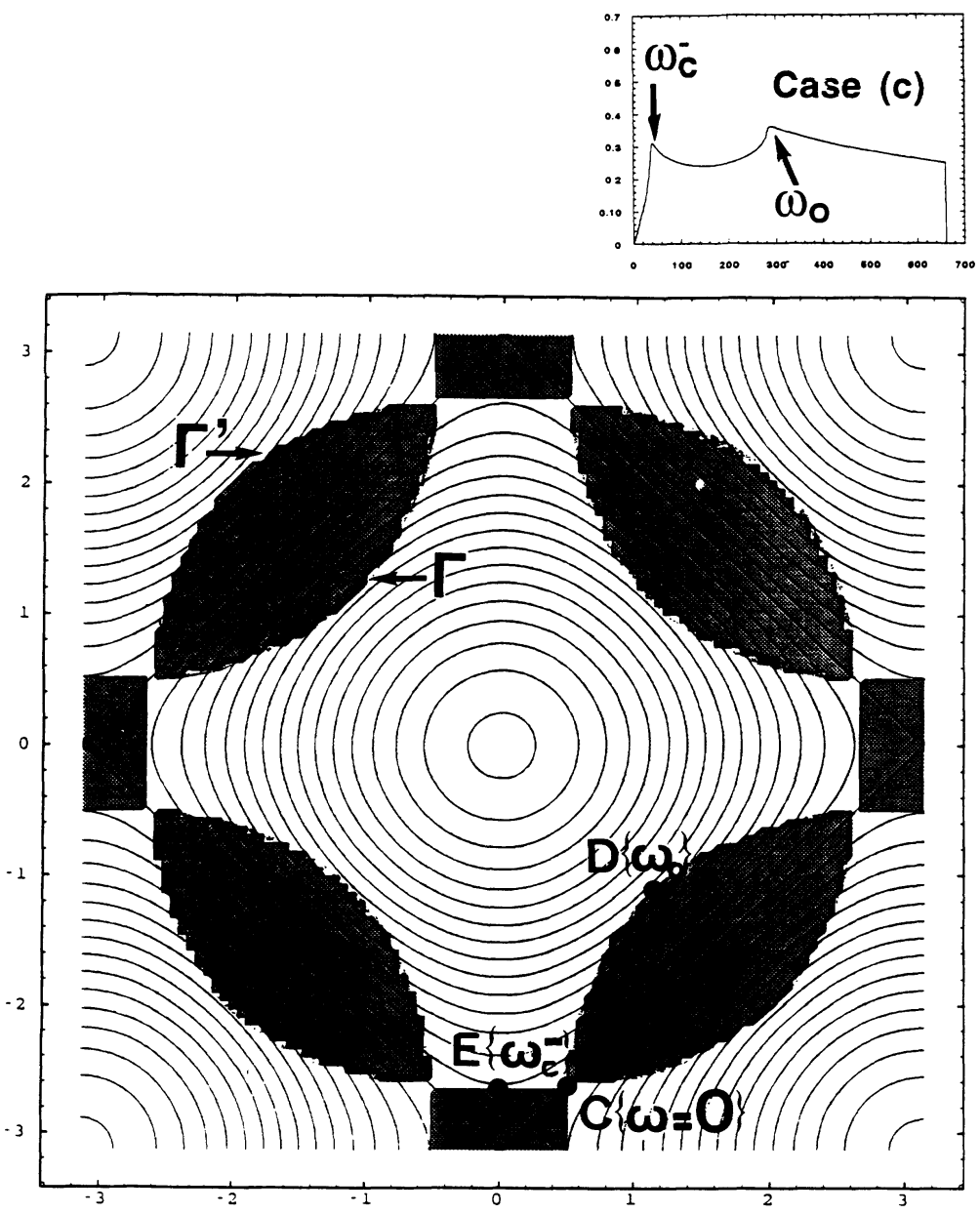

FIG. 7. (Continued). 
version of the $t-t^{\prime}$ Hubbard model considered here.

We will conclude this section on the normal state by studying the temperature dependence of the Knight shift. It is calculated from

$$
\chi^{\prime}(0,0)=\beta \int \frac{\rho_{0}(\xi) d \xi}{4 \cosh ^{2}[\beta(\xi-\mu) / 2]}
$$

The results are reported in Fig. 8 for different values of $\mu$. The high-doping case corresponds to higher values of the Knight shift as expected from simple arguments on the density of states. This feature is in agreement with NMR experiments and makes the $t-t^{\prime}$ model a valuable starting point from a perturbative point of view. Let us remember that the simplest nearest-neighbor model (with only the $t$ term) predicts an opposite order coming from the position of the Van Hove singularity at the middle of the band. Figure 8 also shows a very characteristic increase of $\chi^{\prime}(0,0)$ when lowering the temperature in the highly doped systems. This behavior, effectively observed in the $x=1.0$ yttrium compounds, is simply due to the proximity of the Van Hove singularity located at $\omega=4 t^{\prime}$.

\section{SUPERCONDUCTING STATE}

In the superconducting state with $d$-wave symmetry for the gap (axial superconductivity), the spectrum of excitations is

$$
\varepsilon_{k}=\sqrt{\left(\xi_{k}-\mu\right)^{2}+\Delta_{k}^{2}}
$$

where $\Delta_{k}=2 \Delta_{0}\left(\cos k_{x}-\cos k_{y}\right)$. The equienergetics of excitations are represented in Fig. 9(a). Close to the original Fermi surface $\Sigma_{F}$, it develops four "superconducting ellipses" centered around nodes at which $\varepsilon_{k}=0$ (intersection of the Fermi surface with the first bisectors). Away from $\Sigma_{F}$, we recover the previously described structure with closed and open orbits. The density of excitations has been calculated, and the results are represented in Fig. 9(b). The low-energy part comes from the ellipse contribution: Its variation is linear at low $\omega$ as expected for axial superconductivity in two dimensions. We found a characteristic triple-peak structure which represents the boundary between the ellipses and the previous structure for the first one and the distance from $\mu$ to the Van Hove singularity at $\omega=4 t^{\prime}$ and respectively, the nearer band edge for the two others. The cutoff is provided by
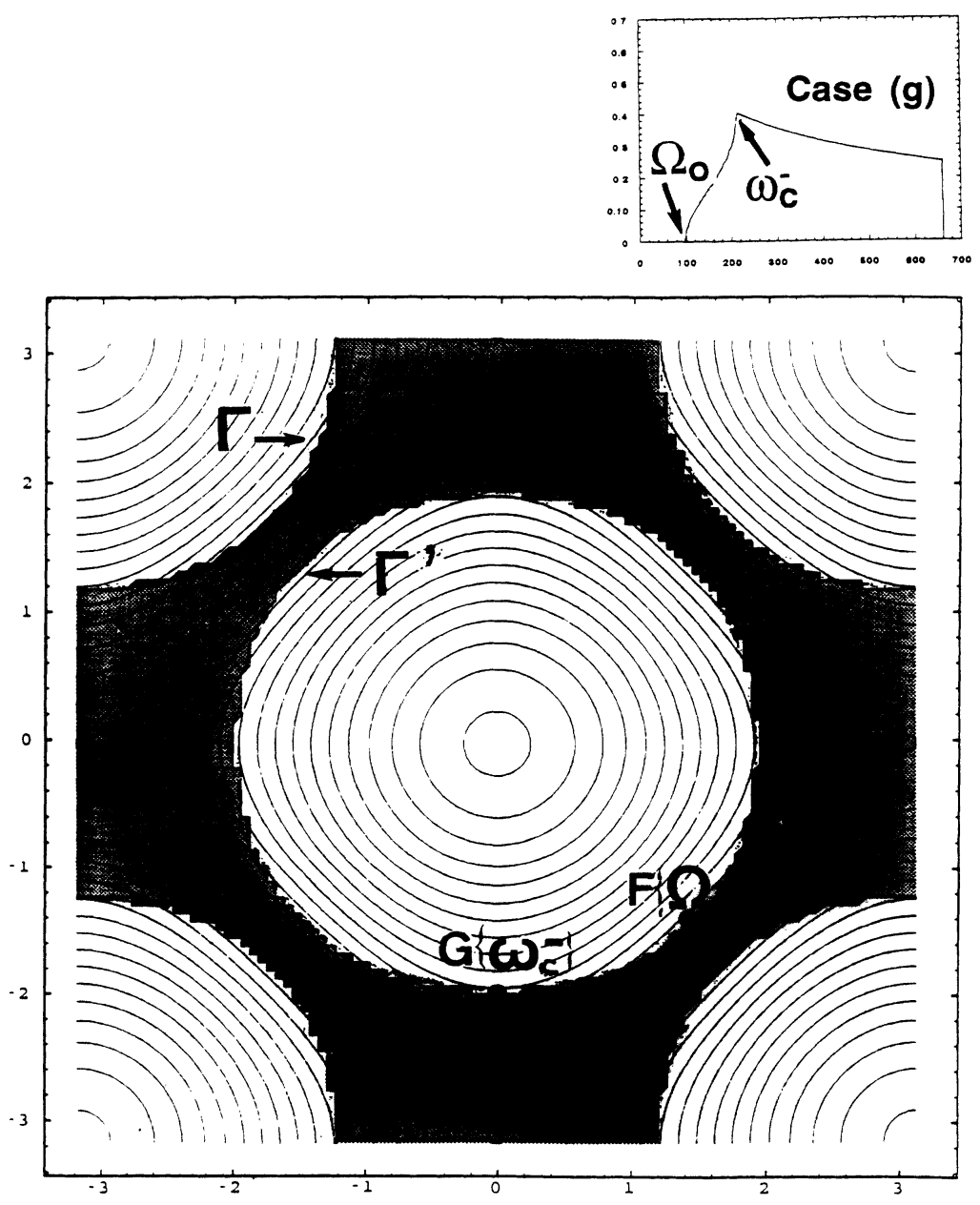

FIG. 7. (Continued). 
TABLE I. Different regimes according to the value of the chemical potential for the frequency dependence of $\chi_{0}^{\prime \prime}(\mathbf{Q}, \omega)$ at $T=0$ without pairing $(\omega>0)$. Values of the gap and cusp positions using the following notation: $\omega_{c}^{+}=2\left(4 t^{\prime}-\mu\right) /\left(1+2 t^{\prime} / t\right)$, $\omega_{c}^{-}=2\left(\mu-4 t^{\prime}\right) /\left(1-2 t^{\prime} / t\right), \quad \omega_{0}=\left(4 t^{2} /-t^{\prime}\right)\left[1-\left(\sqrt{1-\mu t^{\prime} / t^{2}}\right)\right]$, and $\Omega_{0}=\left(4 t^{2} /-t^{\prime}\right)\left[\left(\sqrt{1-\mu t^{\prime} / t^{2}}\right)-1\right]$.

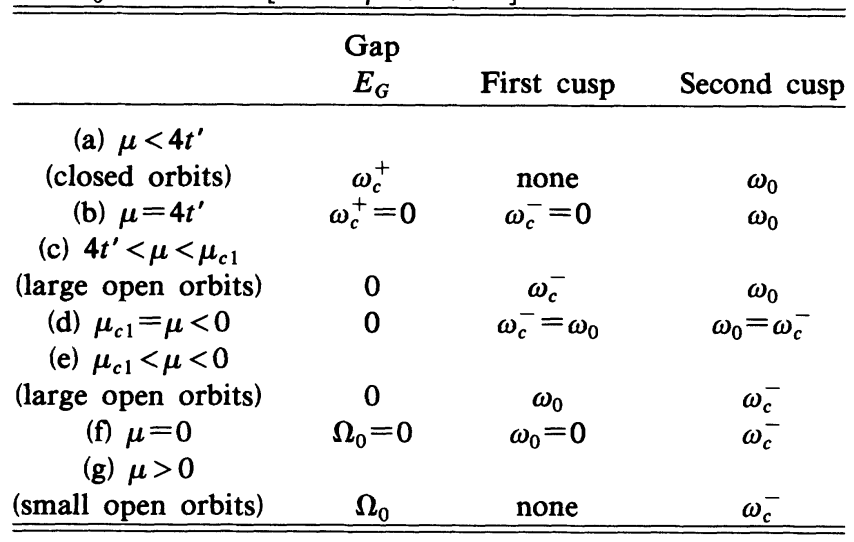

the distance for $\mu$ to the farther band edge.

The unrenormalized dynamic susceptibility is given by (for $\omega>0$ )

$$
\begin{aligned}
& \chi_{0}(\mathbf{q}, \omega)=\sum_{k} \frac{1}{4}\left[1-\frac{\xi_{k} \xi_{k+q}+\Delta_{k} \Delta_{k+q}}{\varepsilon_{k} \varepsilon_{k+q}}\right] \\
& \times \frac{1-n_{F}\left(\varepsilon_{k}\right)-n_{F}\left(\varepsilon_{k+q}\right)}{\omega-\varepsilon_{k}-\varepsilon_{k+q}+i 0^{+}} \\
&+ \frac{1}{2}\left(1+\frac{\xi_{k} \xi_{k+q}+\Delta_{k} \Delta_{k+q}}{\varepsilon_{k} \varepsilon_{k+q}}\right) \\
& \times \frac{n_{F}\left(\varepsilon_{k+q}\right)-n_{F}\left(\varepsilon_{k}\right)}{\omega-\varepsilon_{k+q}+\varepsilon_{k}+i 0^{+}} .
\end{aligned}
$$

The result for the frequency dependence of $\chi_{0}^{\prime \prime}(\mathbf{Q}, \omega)$ at zero temperature is reported in Fig. 10 for the same values of $\mu$ as in the normal phase and for $\Delta_{0}=0.008 t$.

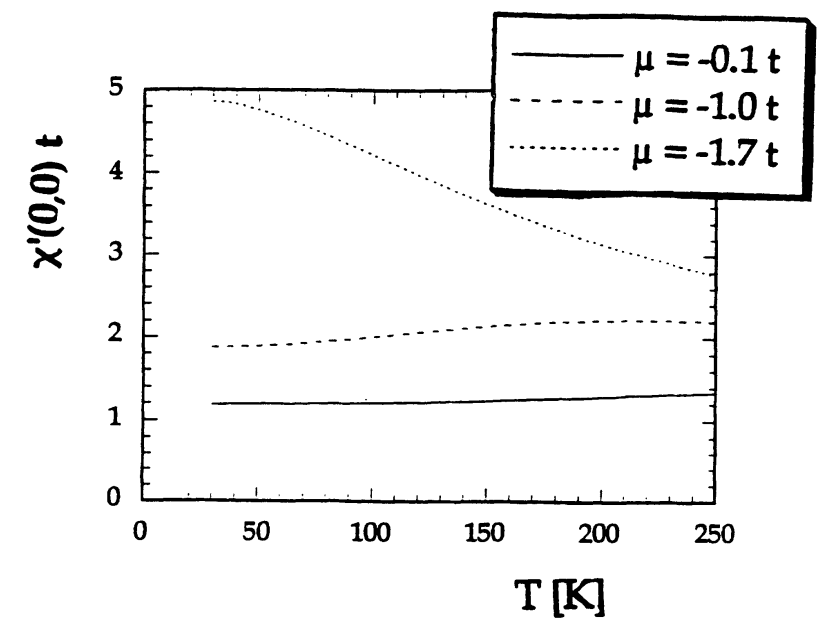

FIG. 8. Uniform static susceptibility without pairing as a function of temperature for different values of $\mu: t=52 \mathrm{meV}$, $t^{\prime}=-0.45 t$.
Fundamental changes are introduced toward the case $\Delta_{0}=0$ without pairing. The most important ones concern the apparition of a threshold in the spectrum of the magnetic excitations whatever the chemical potential is and the existence of a well-defined resonance. As in the normal phase, we propose here a geometrical interpretation of the results. At zero temperature, only the first term in Eq. (12) (creation of pairs of quasiparticles) is allowed coming from the effect of the Fermi factor. Instead of the spectrum of equienergetics $\xi_{k}-\xi_{k+Q}=$ const, we have now to refer to $\varepsilon_{k}+\varepsilon_{k}+Q=$ const associated with the density of pairs of excitations. On the other hand, the coherence factor $\frac{1}{4}\left[1-\left(\xi_{k} \xi_{k+q}+\Delta_{k} \Delta_{k+q}\right) / \varepsilon_{k} \varepsilon_{k+q}\right]$ plays a role very similar to the Fermi factor in the normal phase. In place of a step function, the coherence factor varies continuously from zero to its maximum value 0.5 with a more or

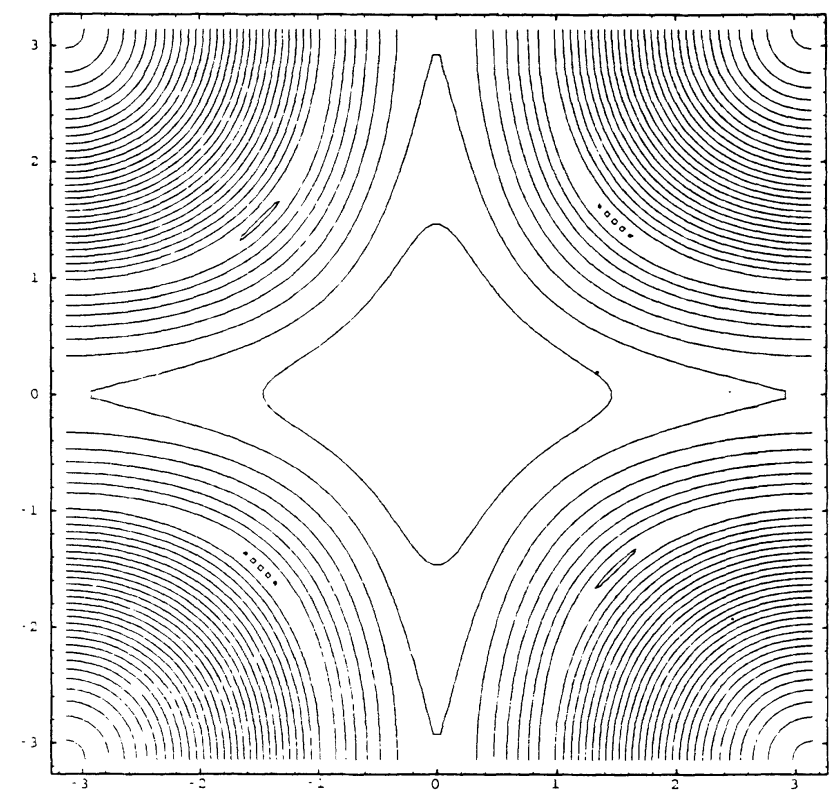

(a)

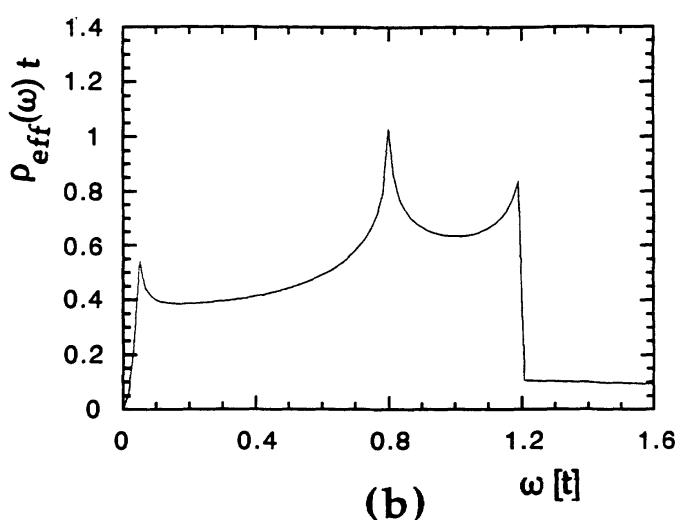

FIG. 9. (a) One-particle excitation energy contours $\varepsilon_{k}=$ const and (b) corresponding density of states in the presence of $d$-wave pairing. Note the formation of four superconducting ellipses around nodes $\left(\varepsilon_{k}=0\right)$ associated with a linear behavior of the density of states at low frequencies. 
less rapid decrease around the previously defined boundaries $\Gamma$ and $\Gamma^{\prime}$ as reported in Fig. 11 as the shaded areas. In the meanwhile, the spectrum of equienergetics $\varepsilon_{k}+\varepsilon_{k+Q}=$ const takes very different shapes depending on the value of the chemical potential, with the formation of ellipses, the center of which moves along the coin vector $\mathbf{A B}$ from the point $A(-\pi / 2,-\pi / 2)$ to $B(0,-\pi)$ when $\mu$ increases from 0 to $4 t^{\prime}$ (cf. Fig. 11). At points $A$ and $B$, respectively, $\left(\varepsilon_{k}+\varepsilon_{k}+Q\right)$ takes the values $\omega_{2}$ and $\omega_{3}$ defined by

$$
\begin{aligned}
& \omega_{2}=2|\mu|, \\
& \omega_{3}=2 \sqrt{\left(4 t^{\prime}-\mu\right)^{2}+\left(4 \Delta_{0}\right)^{2}} .
\end{aligned}
$$

Its value $\omega_{1}$ at the center $O$ of the ellipses [giving the minimum of $\left.\left(\varepsilon_{k}+\varepsilon_{k+Q}\right)\right]$ is evaluated as

$$
\omega_{1}=8 \Delta_{0} \sqrt{\left|\mu / 4 t^{\prime}\right|-\left(\Delta_{0} / 4 t^{\prime}\right)^{2}} .
$$

Table II summarizes the order of the frequencies of $\omega_{1}$, $\omega_{2}$, and $\omega_{3}$ in the different regimes, as well as the value of the gap $E_{G}$ in the spectrum of excitations at $\mathbf{Q}$. It is instructive to compare this table to Table I obtained before in the case without pairing. The new point here is that

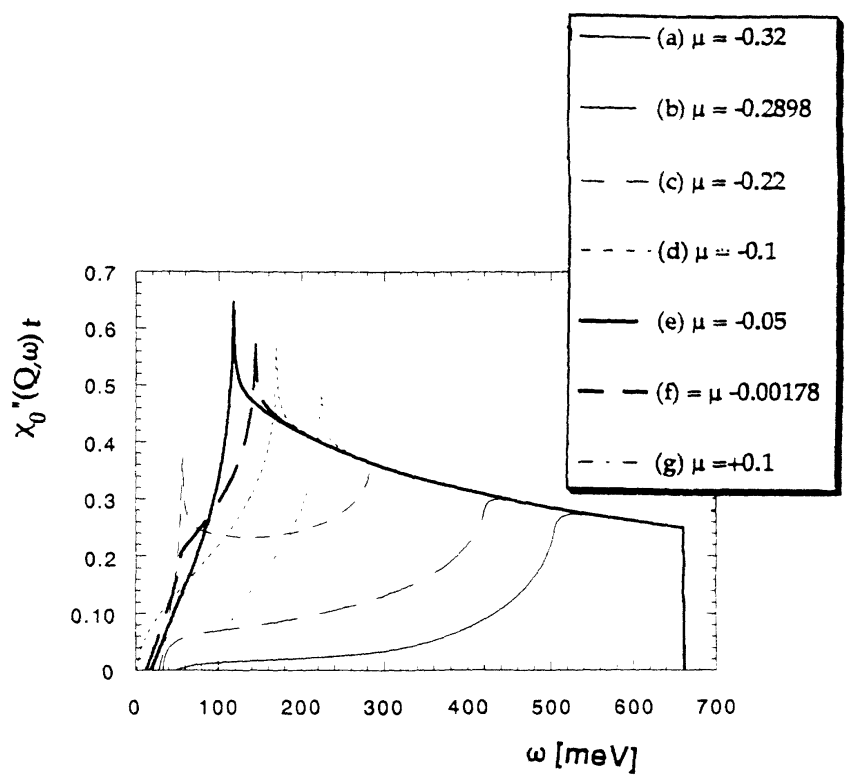

FIG. 10. Spectral weight $\chi_{0}^{\prime \prime}(\mathbf{Q}, \omega)$ vs frequency $\omega$ with $d$ wave pairing at zero temperature: $t=75 \mathrm{meV}, t^{\prime}=-0.45 t$, and $\Delta_{0}=4 \mathrm{meV}$. The enumeration of curves is in accordance with Table II.
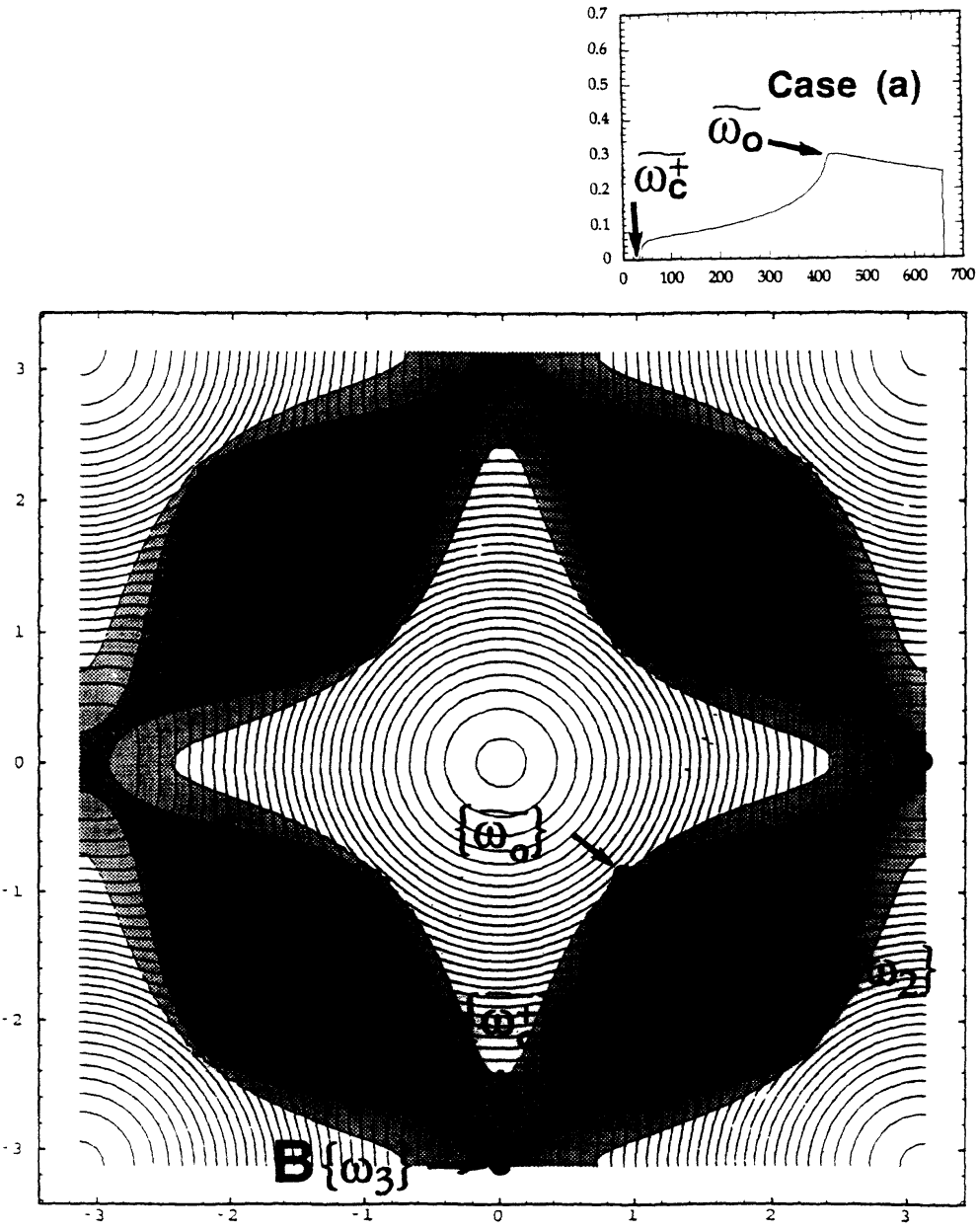

FIG. 11. Equienergetics $\varepsilon_{k+Q}+\varepsilon_{k}=$ const with $d$-wave pairing for the same parameters as in Fig. 10. (a) case (a) $\mu<4 t^{\prime}+2 \Delta_{0}^{2} / t^{\prime}$. (b) case (c) $4 t^{\prime}+2 \Delta_{0}^{2} / t^{\prime}<\mu<\mu_{c 2}$. (c) case (e) $\mu_{c 2}<\mu<2 \Delta_{0}^{2} / t^{\prime}$. (d) case (g) $\mu>2 \Delta_{0}^{2} / t^{\prime}$. The contribution of the shaded region is now reduced by the coherence factor. The convention is dark grey for a coherence factor of less than 0.025 , light grey between 0.025 and 0.25 , and white between 0.25 and 0.5 . In the inset, spectral weight $\chi_{0}^{\prime \prime}(\mathbf{Q}, \omega)$ vs frequency in each case.

(a) 
there is a finite gap in $\chi_{0}^{\prime \prime}(Q, \omega)$ whatever the chemical potential is. Moreover, let us note the analogy which exists between the four regimes (a), (c), (e), and (g) of the case without pairing corresponding, respectively, to $E_{G}=\omega_{c}^{+}, 0,0$, and $\Omega_{0}$ and the new four regimes (a), (c), (e), and (g) obtained in the superconducting case corresponding, respectively, to $E_{G}=\omega_{c}^{+}, \omega_{1}, \omega_{1}$, and $\widetilde{\Omega}_{0}$. The other novelty is the formation of a resonance which is analyzed as a Kohn anomaly of the second kind in the Cooper channel, i.e., equivalently [following our discussion given after Eq. (9)] as the proximity of a Van Hove singularity in the density of double excitations arising from $\delta\left(\omega-\varepsilon_{k}-\varepsilon_{k}+Q\right)$. The resonance is found to occur at a typical value $\widetilde{\omega}_{c}^{-}$, which is nothing but the direct continuation of the cusp value $\omega_{c}^{-}$obtained in the case without pairing [value of $\left(\varepsilon_{k}+\varepsilon_{k+Q}\right)$ at the same point of the Brillouin zone]. The resonance appears as soon as $\mu \geq 4 t^{\prime}+2 \Delta_{0}^{2} / t^{\prime}$ and is progressively shaded off when $\mu$ becomes larger than $2 \Delta_{0}^{2} / t^{\prime}$. We will finish this discussion on the behavior of $\chi_{0}^{\prime \prime}(\mathbf{Q}, \omega)$ in the $d$-wave superconducting phase by two remarks.

(i) Coming from the displacement with doping of the minimum of $\left(\varepsilon_{k}+\varepsilon_{k}+Q\right)$ along the coin vector $\mathbf{A B}$ from
$A$ (associated with $\omega_{2}$ ) to $B$ (associated with $\omega_{3}$ ), we find a characteristic increase of $E_{G}$ when $|\mu|$ increases, as sketched in Fig. 12. It is an interesting result that $E_{G} / 8 \Delta_{0}$ reaches the value 1 only at high doping. At lower doping the gap of excitations is smaller, reaching a value of $2|\mu|$ when the chemical potential goes to zero. This very typical evolution of $E_{G} / 8 \Delta_{0}$ from $|\mu| / 4 \Delta_{0}$ to 1 when doping increases is characteristic of $d$-wave pairing in the frame of the $t-t^{\prime}$ structure considered here. The result would have been completely different in the case of a uniform superconductivity (gap $E_{G}$ of value $2 \sqrt{\mu^{2}+\Delta_{0}^{2}}$ ) or in that of $d$-wave pairing in the simple $t$ structure (gap $E_{G}$ of value $\left.2|\mu|\right)$. This fact has obviously to be brought together with the neutron-scattering results obtained in $\mathrm{YBa}_{2} \mathrm{Cu}_{3} \mathrm{O}_{6+x}$ on the variation of $E_{G}$ with $x$. Let us remark that our result is established for a given $\Delta_{0}$ independent of doping. A complete discussion would consider the variation of $\Delta_{0}$ with doping: This will be done in our forthcoming paper in the frame of the $t-t^{\prime}-J$ model in which the resolution of the gap equation leads to a doping dependence of the gap. Our conclusions will be that the consideration of these additional self-consistent effects would scarcely change the general trend drawn
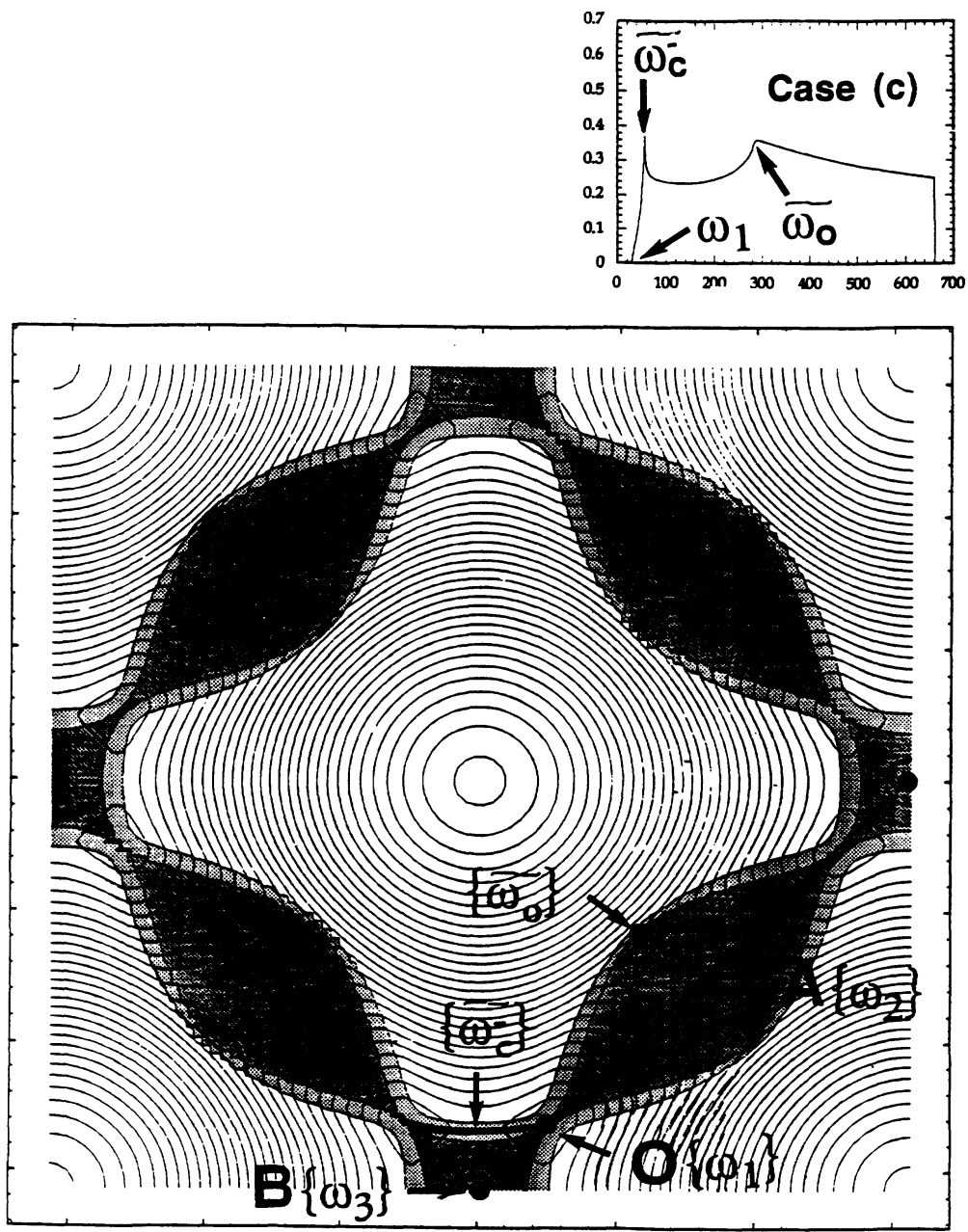

FIG. 11. (Continued). 
above concerning the evolution of $E_{G}$ with doping coming from the displacement of the minimum of $\left(\varepsilon_{k}+\varepsilon_{k+Q}\right)$ along the coin vector $\mathbf{A B}$.

(ii) The same study has been pursued at finite temperatures. The results are reported in Fig. 13 for $\beta=150$ with a temperature dependence of the superconducting gap as represented in Fig. 14 (resulting from the resolution of the gap equation). The effect of temperature is to fill up the spin gap in the intermediate-doping regime $4 t^{\prime}<\mu<0$ and gives back the previously defined values $\omega_{c}^{+}$and $\Omega_{0}$ in the normal phase in the two extreme limits $\mu<4 t^{\prime}$ and $\mu>0$. The resonance is also found to be shaded off with temperature. A convenient way to visualize the temperature effect is to represent the temperature dependence of $\chi_{0}^{\prime \prime}\left(\mathbf{Q}, \omega_{0}\right) / \omega_{0}$ at a given value of the frequency $\omega_{0} \ll E_{G}$ exactly as the experimentalists are used to. The results reported in Fig. 15 show very different behavior depending on the doping. The position $T_{m}$ of the maximum of $\chi_{0}^{\prime \prime}\left(\mathbf{Q}, \omega_{0}\right) / \omega_{0}$ as a function of temperature can be used as a criterion for the temperature scale at which the gap fills up. When $\mu$ moves from $4 t^{\prime}$ to 0 (associated with the regular decrease of $E_{G}$ described above), we find a decrease of $T_{m}$ starting from $T_{c}$. The experimental neutronscattering experiments carried out on $\mathrm{YBa}_{2} \mathrm{Cu}_{3} \mathrm{O}_{6+x}$ show an opposite variation of $T_{m}$ with $x$. There, again, let us outline that all our results are obtained for a given $\Delta_{0}(T)$ variation independent of doping. More realistic models considered in our forthcoming paper will introduce a variation of the superconducting gap with doping and will give rise to rather different predictions for the variation of $T_{m}$. Let us note the presence of a peak structure of $\chi_{0}^{\prime \prime}\left(\mathbf{Q}, \omega_{0}\right) / \omega_{0}$ at $T_{c}$ which is closely related to the Hebel-Schlichter anomaly of $\chi_{0}^{\prime \prime}(0,0)$ somehow washed out by the anisotropic effects brought by $d$-wave pairing.

The renormalization of the bare susceptibility has been carried out within random-phase-approximation (RPA) scheme following

$$
\chi^{\prime \prime}(\mathbf{q}, \omega)=\frac{\chi_{0}^{\prime \prime}(\mathbf{q}, \omega)}{\left[1-U \chi_{0}^{\prime}(\mathbf{q}, \omega)\right]^{2}+\left[U \chi_{0}^{\prime \prime}(\mathbf{q}, \omega)\right]^{2}} .
$$

The result at zero temperature is reported in Fig. 16 for a given choice of the parameters, $t=75 \mathrm{meV}, t^{\prime}=-0.1 t$, $\mu=-14.48 \mathrm{meV}$, and $\Delta_{0}=1.45 \mathrm{meV}$, corresponding to
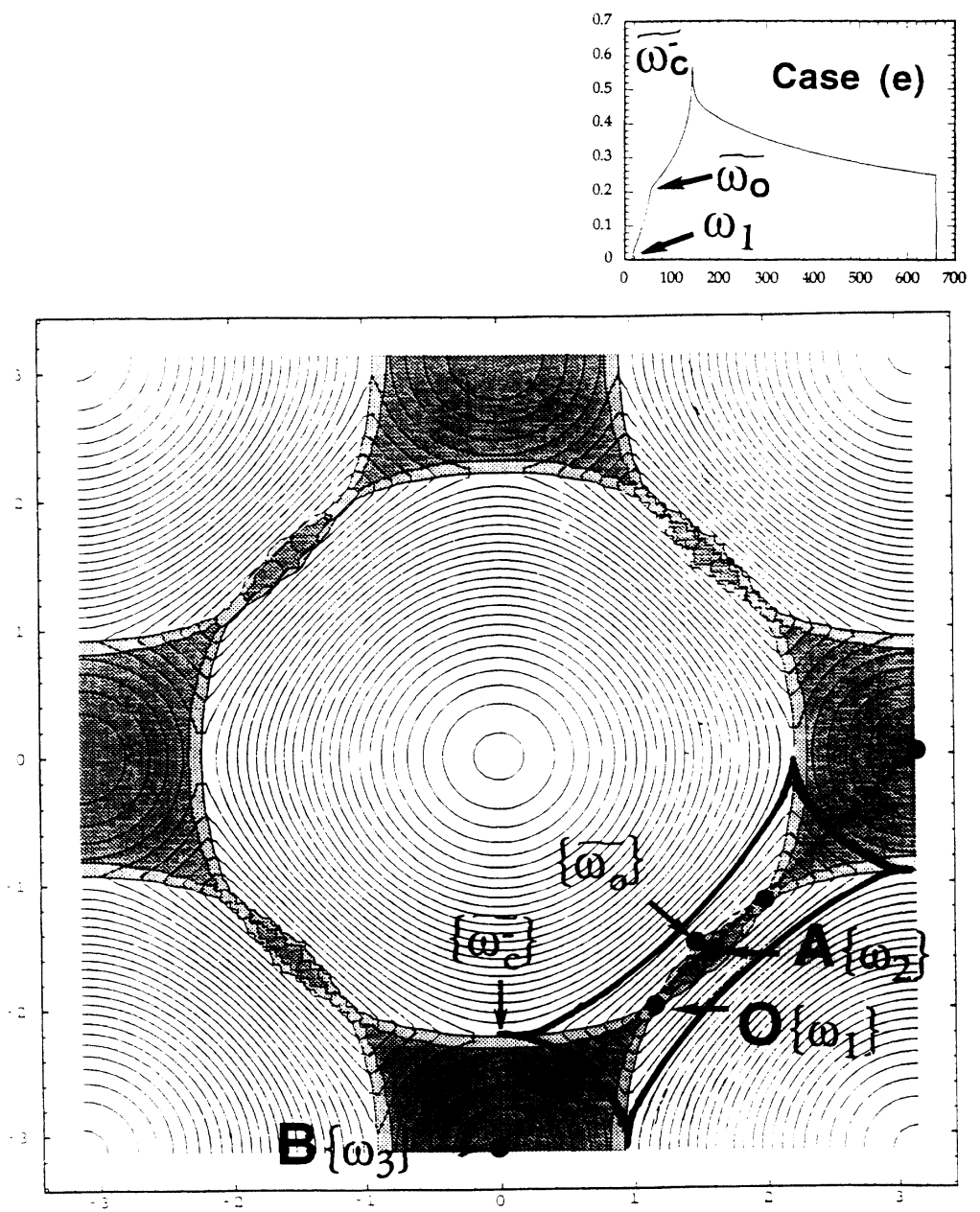

FIG. 11. (Continued). 
regime (e) of Table II. The frequency dependence that we get for $\chi^{\prime \prime}(\mathbf{Q}, \omega)$, bears striking resemblance to the neutron-scattering results reported by some authors ${ }^{2}$ in $\mathrm{YBa}_{2} \mathrm{Cu}_{2} \mathrm{O}_{6.92}$ at $5 \mathrm{~K}$ and represented in the inset of the same figure. One notes the presence of a gap of order 28 $\mathrm{meV}$ (analytically given by $\omega_{1}$ ) followed by a plateau starting from $30 \mathrm{meV}$ and a resonance around $41 \mathrm{meV}$. The characteristic frequencies for both the plateau and resonance are, respectively, given by $\widetilde{\omega}_{0}$ and $\widetilde{\omega}_{c}^{-}$. The former corresponds to the cusp already present in the frequency dependence of $\chi_{0}^{\prime \prime}(\mathbf{Q}, \omega)$ reported in Fig. 10; the latter owes its origin to the existence of the four superconducting ellipses in the spectrum of excitations $\varepsilon_{k}$, giving rise to a Van Hove-type singularity for the pairexcitation spectrum $\left(\varepsilon_{k}+\varepsilon_{k+Q}\right)$. This very typical behavior emerges from the effects of a double anisotropy, that of the superconducting gap (axial for $d$-wave superconductivity) and that of the Fermi surface, according to photoemission measurements and modeled here in our $t$ $t^{\prime}$ model. We will see in our next paper that this feature will survive in the strong-coupling regime.

Finally, the Knight shift has been calculated according to

$$
\chi^{\prime}(0,0)=\beta \int \frac{\rho_{0}(\xi) d \xi}{4 \cosh ^{2}(\beta \varepsilon / 2)} .
$$

The results reported in Fig. 17 show a Yosida-like behavior for the Knight shift with a linear temperature dependence at very low temperatures (instead of exponential for uniform superconductivity) as expected for axial superconductivity. This is directly related to the linear frequency dependence of the density of excitations at low frequencies [see Fig. 9(b)] when the four ellipses (superconducting ellipses) develop around nodes of the superconducting gap.

This behavior may well account for the NMR observations in the surdoped $\mathrm{YBa}_{2} \mathrm{Cu}_{3} \mathrm{O}_{7.0}$ system. However, the approach is less successful for heavily doped systems since it predicts a reduction of the Knight shift only below $T_{c}$ in contradiction with the experimental results which show a regular decrease of the Knight shift initialized from well above $T_{c}$. This failure gives strong arguments in favor of the strong-coupling regime. It will be tackled in our next paper in which the discussion is set off from the point of view of the formation of a singlet-RVB state in the temperature range $T_{c}<T<T_{\mathrm{RVB}}$.
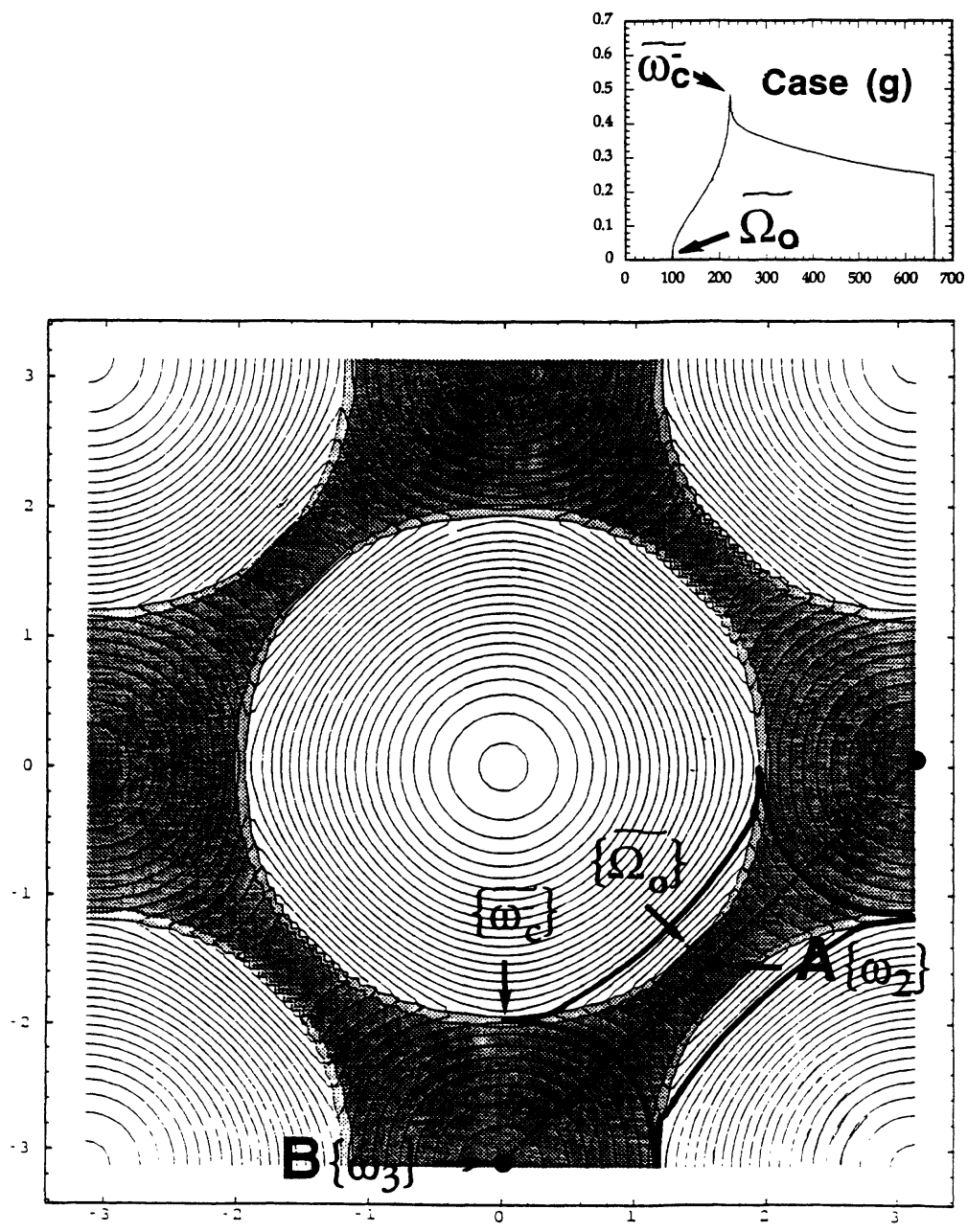

FIG. 11. (Continued). 
TABLE II. Different regimes according to the value of the chemical potential for the frequency dependence of $\chi_{0}^{\prime \prime}(\mathbf{Q}, \omega)$ at $T=0$ with $d$-wave pairing $(\omega>0): \omega_{1}=8 \Delta_{0} \sqrt{\mu / 4 t^{\prime}-\left(\Delta_{0} / 2 t^{\prime}\right)^{2}}, \omega_{2}=2|\mu|$, and $\omega_{3}=2 \sqrt{\left(4 t^{\prime}-\mu\right)^{2}+\left(4 \Delta_{0}\right)^{2}}$. Values of the gap and cusp (if any) using the following notation: $\widetilde{\omega}_{c}^{+}=\varepsilon_{k}+\varepsilon_{k+Q} \quad$ at $\quad \mathbf{k}=\left(k_{1}, 0\right), \quad k_{1}=\arccos \left[(\mu+2 t) / 2\left(t+2 t^{\prime}\right)\right], \quad \widetilde{\omega}_{c}^{-}=\varepsilon_{k}+\varepsilon_{k+Q} \quad$ at $\quad \mathbf{k}=\left(k_{2}, 0\right)$, $k_{2}=\arccos \left[(\mu-2 t) / 2\left(t-2 t^{\prime}\right)\right], \quad \widetilde{\omega}_{0}=\varepsilon_{k}+\varepsilon_{k+Q} \quad$ at $\quad \mathbf{k}=\left(k_{3}, k_{3}\right), \quad k_{3}=\arccos \left\{\left[t /-2 t^{\prime}\right][1\right.$ $\left.\left.-\sqrt{1-\mu t^{\prime} / t^{2}}\right]\right\}, \widetilde{\Omega}_{0}=\varepsilon_{k}+\varepsilon_{k+Q}$ at $\mathbf{k}=\left(k_{4}, k_{4}\right), k_{4}=\arccos \left\{\left[t /-2 t^{\prime}\right]\left[1-\sqrt{\mu t^{\prime} / t^{2}-1}\right]\right\}$.

\begin{tabular}{|c|c|c|c|c|}
\hline & $\begin{array}{l}\text { Increasing } \\
\text { order }\end{array}$ & $\begin{array}{c}\text { Gap } \\
E_{G}\end{array}$ & Resonance & Cusp \\
\hline (a) $\mu<4 t^{\prime}+2 \frac{\Delta_{0}^{2}}{t^{\prime}}$ & $\omega_{3}<\omega_{2}$ & $\widetilde{\omega}_{c}^{+}>\omega_{3}$ & none & $\widetilde{\omega}_{0}$ \\
\hline (b) $\mu=4 t^{\prime}+2 \frac{\Delta_{0}^{2}}{t^{\prime}}$ & $\omega_{1}=\omega_{3}<\omega_{2}$ & $\omega_{1}=\omega_{3}$ & $\widetilde{\omega}_{c}^{-}$ & $\widetilde{\omega}_{0}$ \\
\hline (c) $4 t^{\prime}+2 \frac{\Delta_{0}^{2}}{t^{\prime}}<\mu<\mu_{c 2}$ & $\omega_{1}<\omega_{3}<\omega_{2}$ & $\omega_{1}$ & $\widetilde{\omega}_{c}^{-}$ & $\widetilde{\omega}_{0}$ \\
\hline (d) $\mu_{c 2}=\mu<2 \frac{\Delta_{0}^{2}}{t^{\prime}}$ & $\omega_{1}<\omega_{3}=\omega_{2}$ & $\omega_{1}$ & $\widetilde{\omega}_{c}^{-}$ & $\widetilde{\omega}_{0}$ \\
\hline (e) $\mu_{c 2}<\mu<2 \frac{\Delta_{0}}{t^{\prime}}$ & $\omega_{1}<\omega_{2}<\omega_{3}$ & $\omega_{1}$ & $\widetilde{\omega}_{c}^{-}$ & $\widetilde{\omega}_{0}$ \\
\hline (f) $\mu=2 \frac{\Delta_{0}^{2}}{t^{\prime}}$ & $\omega_{1}=\omega_{2}<\omega_{3}$ & $\omega_{1}=\omega_{2}$ & $\widetilde{\omega}_{c}^{-}$ & $\widetilde{\omega}_{0}$ \\
\hline (g) $\mu>2 \frac{\Delta_{0}^{2}}{t^{\prime}}$ & $\omega_{2}<\omega_{3}$ & $\widetilde{\Omega}_{0}>\omega_{2}$ & $\widetilde{\omega}_{c}^{-}$ & none \\
\hline
\end{tabular}

\section{CONCLUSIONS}

To conclude, let us summarize the essential results established in this paper for the $t-t^{\prime}$-lattice Hubbard model in the weak-coupling limit.

In the normal phase, coming from the Van Hove singularity in the density of states located at $\omega=4 t^{\prime}$ (cf. Fig. 2), we predict the correct order for the Knight shift with doping (cf. Fig. 8) in fair agreement with the experimental results obtained in $\mathrm{YBa}_{2} \mathrm{Cu}_{3} \mathrm{O}_{6+x}$. For the dynamic susceptibility $\chi_{0}^{\prime \prime}(\mathbf{Q}, \omega)$, one gets three regimes.

(i) $\mu<4 t^{\prime}$ (closed orbits): the frequency dependence of $\chi_{0}^{\prime \prime}(\mathbf{Q}, \omega)$ at zero temperature shows a gap followed by a cusp at higher frequency.

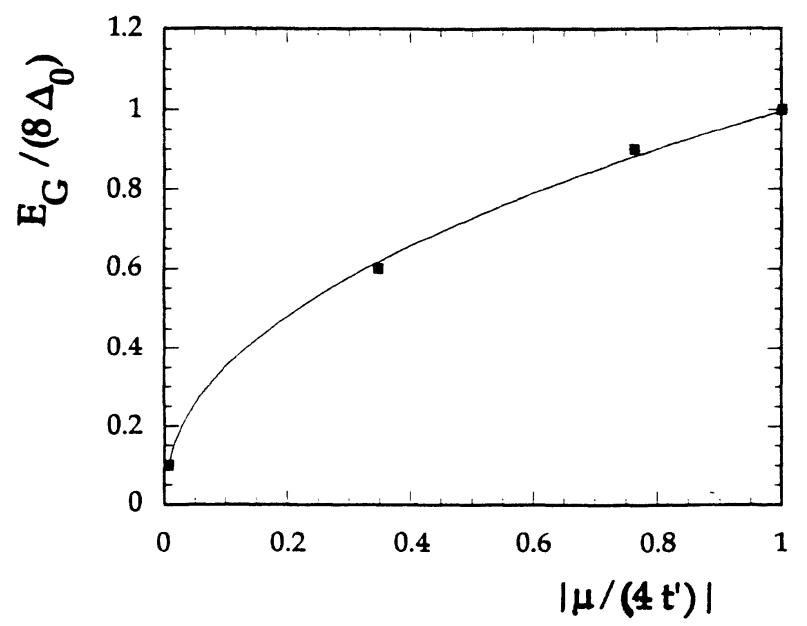

FIG. 12. Spin gap in the excitation spectrum of $\chi_{0}^{\prime \prime}(\mathbf{Q}, \omega)$ for $d$-wave pairing vs chemical potential (both in renormalized units). The points are taken from numerical calculations; the solid line is drawn from the analytical expression of $\omega_{1}$. (ii) $4 t^{\prime}<\mu<0$ (large open orbits): there is no spin gap but instead a typical double-cusp structure.

(iii) $\mu>0$ (small open orbits): the spectrum of excitations has again a threshold with a cusp at higher frequency.

The behavior in each regime is summarized in Table I and sketched in Fig. 3. A geometrical construction has been given in Fig. 7 to understand the different features of the spectrum of excitations. In the current regime of interest $\left(4 t^{\prime}<\mu<0\right)$, that is to say for reasonable values of doping and choices of the ratio $t^{\prime} / t$, we find no spin gap,

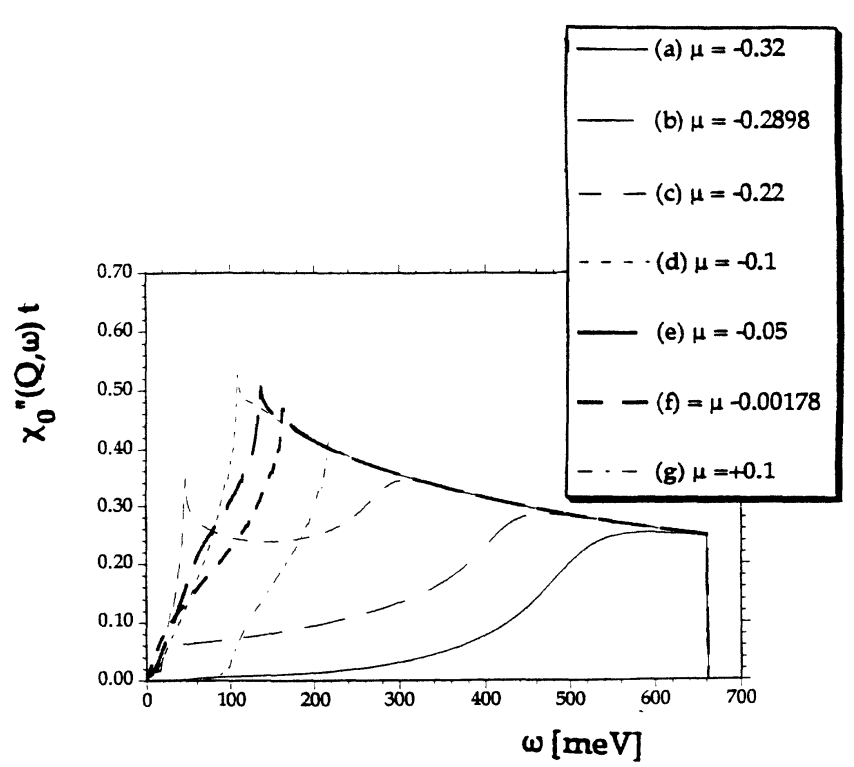

FIG. 13. Spectral weight $\chi_{0}^{\prime \prime}(\mathbf{Q}, \omega)$ vs frequency $\omega$ with $d$ wave pairing at $100 \mathrm{~K}$. The parameters are the same as in Fig. 10. The enumeration of curves is in accordance with Table II. 


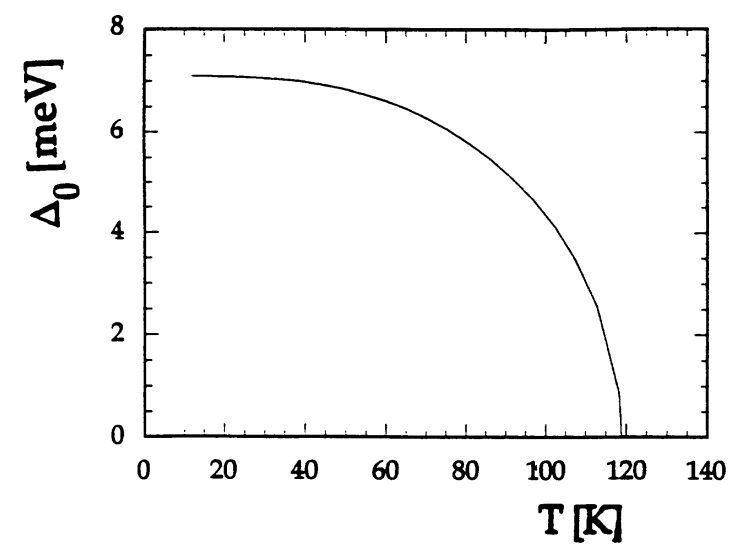

FIG. 14 Pairing parameter $\Delta_{0}$ as a function of temperature. The pairing parameter is obtained as a solution of the gap equation. The interaction parameter is chosen such that $T_{c}$ is of the order of $120 \mathrm{~K}$.

in contradiction with the neutron-scattering results in yttrium compounds reported by some of the groups. We will propose in our forthcoming paper an alternative to reconcile the information coming from photoemission (shape of the Fermi surface) and neutron-diffraction experiments in terms of the formation of a RVB-singlet state in the strong-coupling limit given by the $t-t^{\prime}-J$ model.

In the superconducting state, we have shown how $d$ wave pairing creates four additional superconducting ellipses in the spectrum of individual excitations as represented in Fig. 9. These ellipses are responsible for a linear $\omega$ dependence of the density of excitations at low frequencies typical of axial superconductivity in two dimensions. Consequently, the Knight shift follows a Yosida-type law with a linear $T$ dependence at very low temperatures (cf. Fig. 17). As regards the dynamic susceptibility $\chi_{0}^{\prime \prime}(\mathbf{Q}, \omega)$ at zero temperature, our calculations

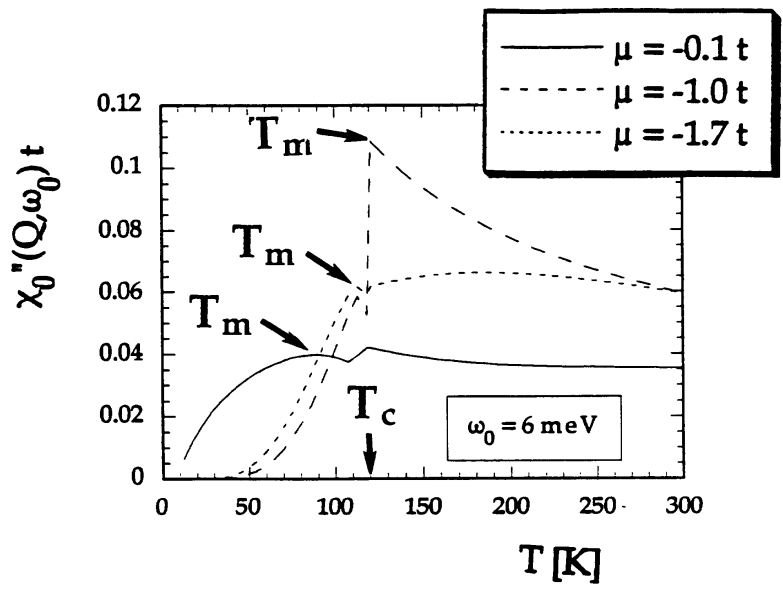

FIG. 15. Temperature dependence of $\chi_{0}^{\prime \prime}\left(\mathbf{Q}, \omega_{0}\right)$ for different $\mu: t=52 \mathrm{meV}, t^{\prime}=-0.45 t$, and $\omega_{0}=6 \mathrm{meV}$. Note the presence of a Hebel-Schlichter-type anomaly at $T_{c} \cdot \chi_{0}^{\prime \prime}\left(\mathbf{Q}, \omega_{0}\right)$ takes its maximum value at $T_{m}<T_{c}$ increasing with $|\mu|$, reaching $T_{c}$ at $\mu=4 t^{\prime}$.
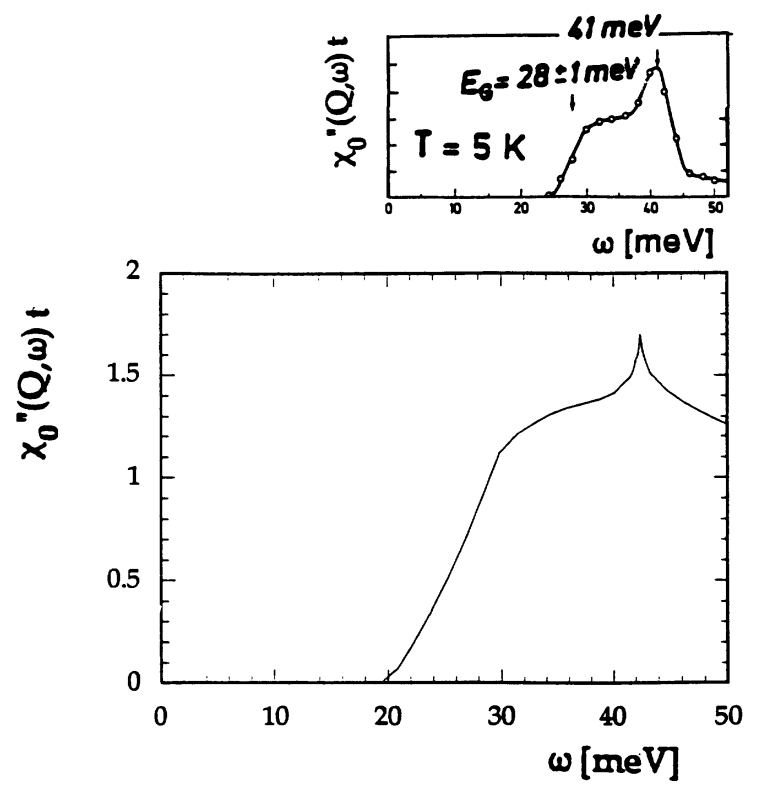

FIG. 16. RPA-renormalized spectral weight $\chi^{\prime \prime}(\mathbf{Q}, \omega)$ vs frequency $\omega$ with $d$-wave pairing at zero temperature for a special choice of parameters: $t=75 \mathrm{meV}, t^{\prime}=-0.1 t, \mu=-14.48 \mathrm{meV}$, and $\Delta_{0}=1.45 \mathrm{meV}$. Note the presence of a resonance with a plateau and a gap reminding one of the neutron-scattering experiments (Ref. 2) for the $\mathrm{YBa}_{2} \mathrm{Cu}_{3} \mathrm{O}_{6.92}$ compound at $5 \mathrm{~K}$ reported in the inset.

(cf. Fig. 10) show a threshold in the spectrum of excitations whatever the chemical potential is, with the onset of a resonance analyzed as a Kohn anomaly of the second kind in the Cooper channel. When properly renormalized through the RPA scheme, the frequency dependence of $\chi_{0}^{\prime \prime}(\mathbf{Q}, \omega)$ (cf. Fig. 16) bears a striking resemblance with the neutron-scattering results established by RossatMignod $e t$ al. in the $x=0.92$ compound at $T=5 \mathrm{~K}$, with a gap structure superimposed on a resonance above the threshold. A geometrical construction is proposed in

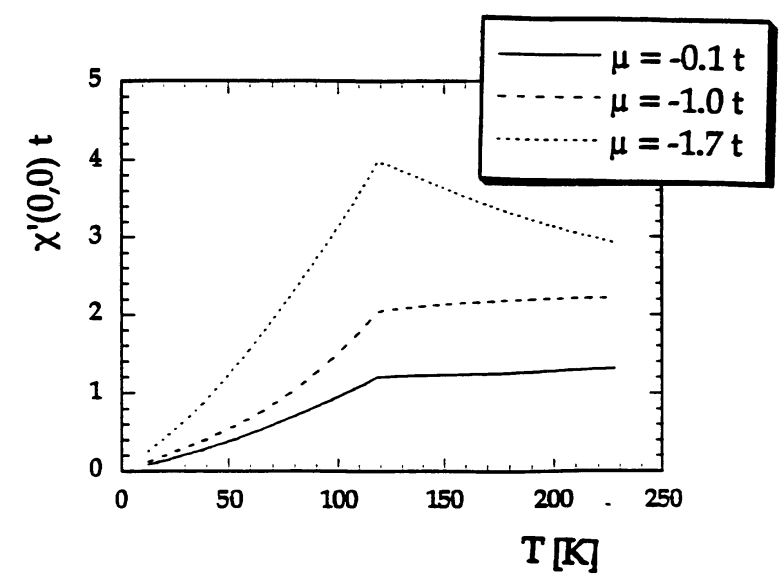

FIG. 17. Uniform static susceptibility with $d$-wave pairing as a function of temperature for different values of $\mu$. The parameters are the same as for Fig. 15. Note the Yosida-like behavior below $T_{c}$ with a linear low-temperature dependence typical of axial superconductivity in two dimensions. 
Fig. 11 to illustrate the results. Coming from the displacement of the minimum of $\left(\varepsilon_{k}+\varepsilon_{k+Q}\right)$ along the coin vector $\mathbf{A B}$, the threshold $E_{G} / 8 \Delta_{0}$ evolves from $|\mu| / 4 \Delta_{0}$ to 1 when $\mu$ goes along from 0 to $4 t^{\prime}$ clearing out the crossover between two different regimes (cf. Fig. 12). Table II recapitulates the corresponding situation. Moreover, the temperature $T_{m}$ at which the gap fills uplocated from the position of the maximum of $\chi_{0}^{\prime \prime}\left(\mathbf{Q}, \omega_{0}\right) / \omega_{0}$ at $\omega_{0}<<E_{G}$ (see Fig. 15) - is found to considerably change in the meanwhile, reaching $T_{c}$ only in highly doped regimes. To our point of view, this feature concerning the combined evolution of $E_{G}$ and $T_{m}$ with doping is bound to play a crucial role in the interpretation of experiments.

\section{ACKNOWLEDGMENTS}

We acknowledge the European Economic Community (EEC) for providing financial support under Contract No. ERB 4050 PL920925. G.S. was supported by EEC Grant No. SCI/915056. This work has benefited from active and stimulating discussions with C. Berthier, L. P. Regnault, and J. Rossat-Mignod who are here kindly acknowledged. We also want to address our best thanks to P. Burlet, D. Grempel, M. Horvatic, and C. Pépin.
${ }^{1}$ T. E. Mason, G. Aeppli, and H. A. Mook, Phys. Rev. Lett. 68, 1414 (1992).

2J. Rossat-Mignod, L. P. Regnault, C. Vettier, P. Burlet, J. Y. Henry, and G. Lapertot, Physica B 169, 58 (1991); J. RossatMignod, L. P. Regnault, C. Vettier, P. Bourges, P. Burlet, J. Bossy, J. Y. Henry, and G. Lapertot, Physica C 185-189, 86 (1991); J. Rossat-Mignod, L. P. Regnault, P. Bourges, P. Burlet, C. Vettier, and J. Y. Henry, in Frontiers in Solid State Sciences, edited by L. C. Gupta and M. S. Multani (World Scientific, Singapore, 1993), Vol. 1, p. 265.

${ }^{3}$ J. M. Tranquada, W. J. L. Buyers, H. Chou, T. E. Mason, M. Sato, S. Shamoto, and G. Shirane, Phys. Rev. Lett. 64, 800 (1990); G. Shirane, J. Als-Nielsen, M. Nielsen, J. M. Tranquada, H. Chou, S. Shamoto, and M. Sato, Phys. Rev. B 41, 6547 (1990); P. Bourges, P. M. Gehring, B. Hennion, A. H. Moudden, J. M. Tranquada, G. Shirane, S. Shamoto, and M. Sato, ibid. 43, 8690 (1991).

${ }^{4}$ P. M. Gehring, J. M. Tranquada, G. Shirane, J. R. D. Copley, R. W. Erwin, M. Sato, and S. Shamoto, Phys. Rev. B 44, 2811 (1991).

${ }^{5}$ H. A. Mook, M. Yethiraj, G. Aeppli, T. E. Mason, and T. Armstrong, Phys. Rev. Lett. 70, 3490 (1993).

${ }^{6}$ M. Horvatic, P. Butaud, P. Segransan, Y. Berthier, C. Berthier, J. Y. Henry, and M. Couach, Physica C 166, 151 (1990); C. Berthier, Y. Berthier, B. Butaud, M. Horvatic, Y. Kitaoka, and P. Segransan, in Dynamics of Magnetic Fluctuations in High- $T_{c}$ Materials, edited by G. Reiter, P. Horsh, and G. Psaltakis (Plenum, New York, 1991), Vol. 246, p. 73.

${ }^{7}$ R. E. Walstedt, W. W. Warren, R. Tycko, R. F. Bell, G. F.
Brennert, R. J. Cava, L. Schneemeyer, and J. Waszczak, Phys. Rev. B 38, 9303 (1988); M. Takigawa, A. P. Reyes, P. C. Hammel, J. D. Tompson, R. H. Heffner, Z. Fisk, and K. C. Ott, ibid. 43, 3698 (1991); P. C. Hammel, M. Takigawa, R. H. Heffner, Z. Fisk, and K. C. Ott, Phys. Rev. Lett. 63, 1992 (1989).

${ }^{8}$ C. M. Varma, P. B. Littlewood, S. Schmitt-Rink, E. Abrahams, and A. E. Ruckenstein, Phys. Rev. Lett. 63, 1996 (1989).

${ }^{9}$ A. J. Millis, H. Monien, and D. Pines, Phys. Rev. B 42, 167 (1990).

${ }^{10}$ N. Bulut and D. J. Scalapino (unpublished).

${ }^{11}$ A. Virosztek and J. Ruvalds, Phys. Rev. B 42, 42 (1990).

${ }^{12}$ S. V. Maleyev, J. Phys. 2, 181 (1992); (unpublished).

${ }^{13}$ T. Tanamoto, K. Kuboki, and H. Kukuyama, J. Phys. Soc. Jpn. 60, 3072 (1991).

${ }^{14}$ D. R. Grempel and M. Lavagna, Solid State Commun. 83, 595 (1992).

${ }^{15}$ W. E. Pickett, R. E. Cohen, and H. Krakauer, Phys. Rev. B 42, 8764 (1990); M. S. Hybertsen, E. B. Stechel, M. Schluter, and D. R. Jennison, ibid. 41, 11068 (1990).

${ }^{16}$ P. Bénard, L. Chen, and A.-M. S. Tremblay (unpublished).

${ }^{17}$ H. Q. Lin and J. E. Hirsch, Phys. Rev. B 35, 3359 (1987); J. H. Kim, K. Levin, and A. Auerbach, ibid. 39, 11633 (1989); D. M. Newns, P. C. Pattnaik, and C. C. Tsuei, ibid. 43, 3075 (1991); H. Chi and A. D. S. Nagi, ibid. 46, 421 (1992); J. Wheatley (unpublished).

${ }^{18}$ G. Stemmann, C. Pépin, and M. Lavagna (unpublished).

${ }^{19}$ P. W. Anderson, Science 235, 1196 (1987); G. Kotliar, Phys. Rev. B 37, 3664 (1988). 


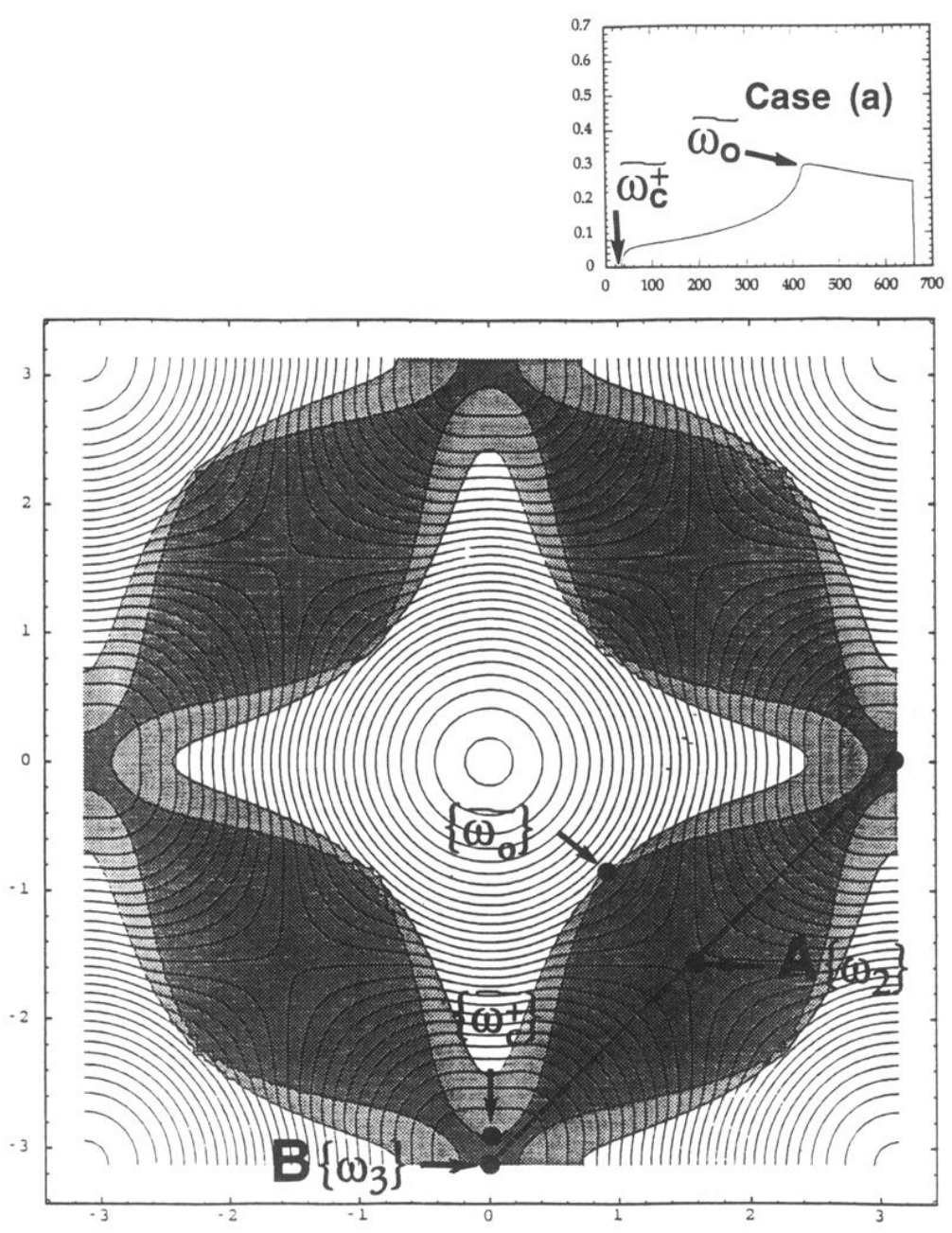

(a)
FIG. 11. Equienergetics $\varepsilon_{k+Q}+\varepsilon_{k}=$ const with $d$-wave pairing for the same parameters as in Fig. 10. (a) case (a) $\mu<4 t^{\prime}+2 \Delta_{0}^{2} / t^{\prime}$. (b) case (c) $4 t^{\prime}+2 \Delta_{0}^{2} / t^{\prime}<\mu<\mu_{c 2}$. (c) case (e) $\mu_{c 2}<\mu<2 \Delta_{0}^{2} / t^{\prime}$. (d) case (g) $\mu>2 \Delta_{0}^{2} / t^{\prime}$. The contribution of the shaded region is now reduced by the coherence factor. The convention is dark grey for a coherence factor of less than 0.025 , light grey between 0.025 and 0.25 , and white between 0.25 and 0.5 . In the inset, spectral weight $\chi_{0}^{\prime \prime}(\mathbf{Q}, \omega)$ vs frequency in each case. 

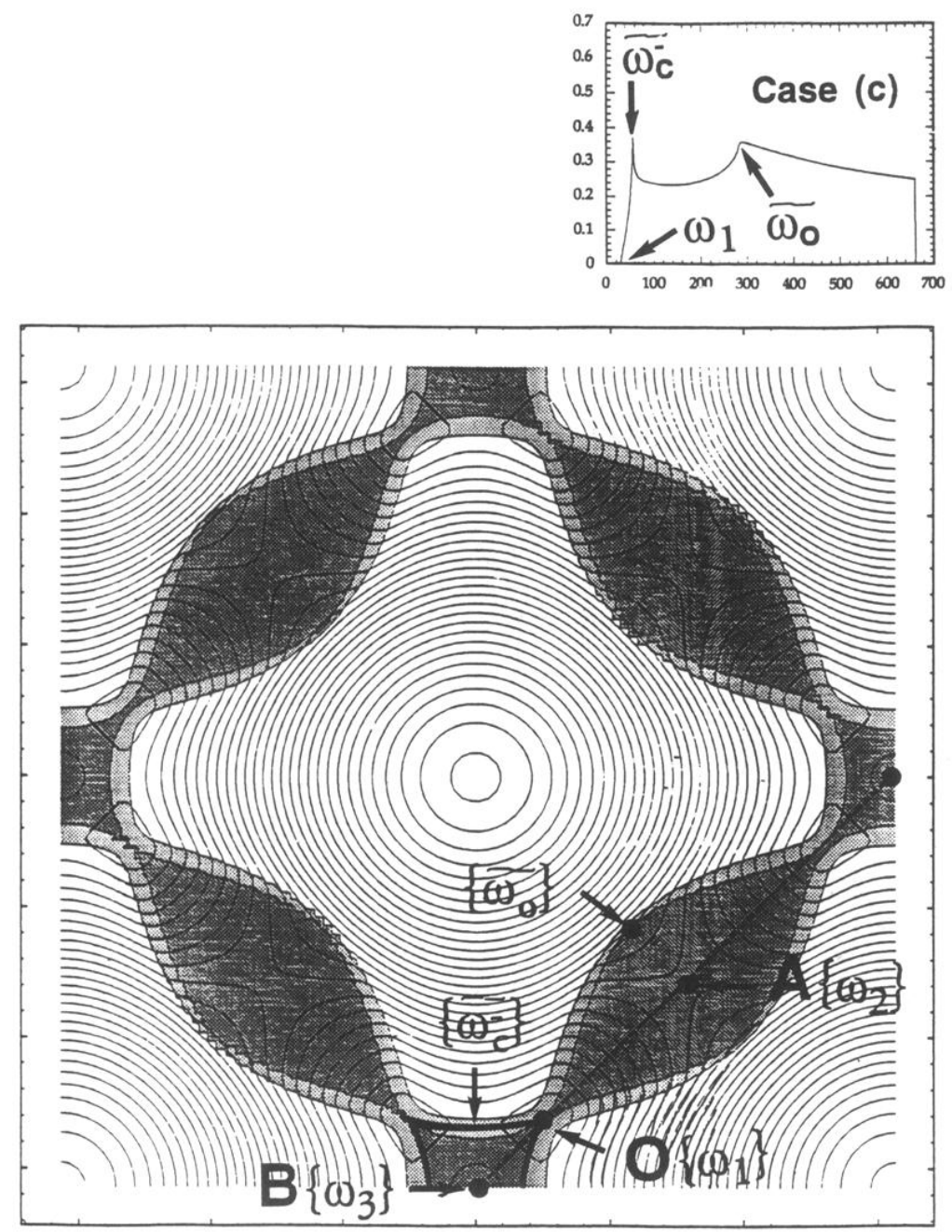

FIG. 11. (Continued).

(b) 

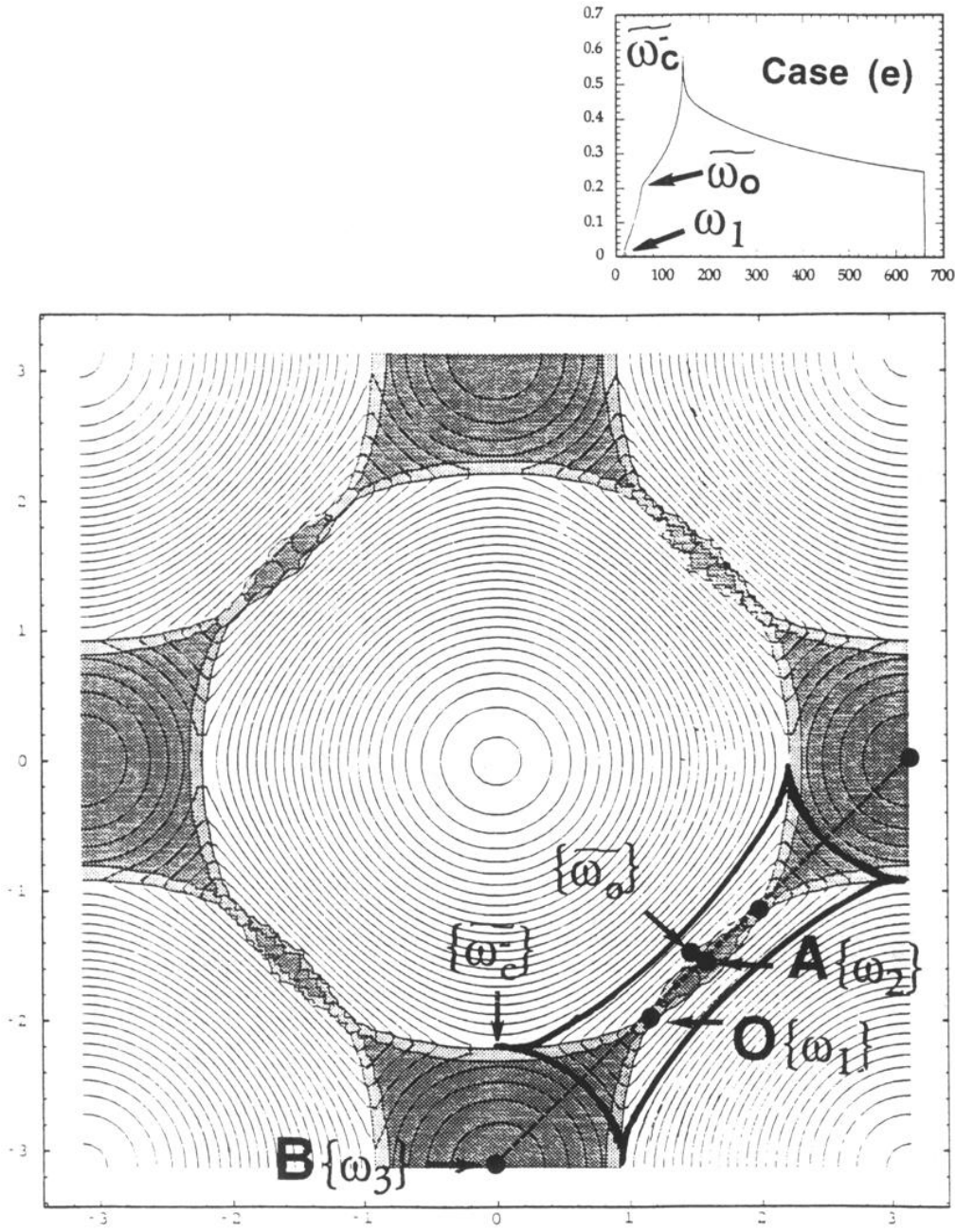

FIG. 11. (Continued).

(c) 

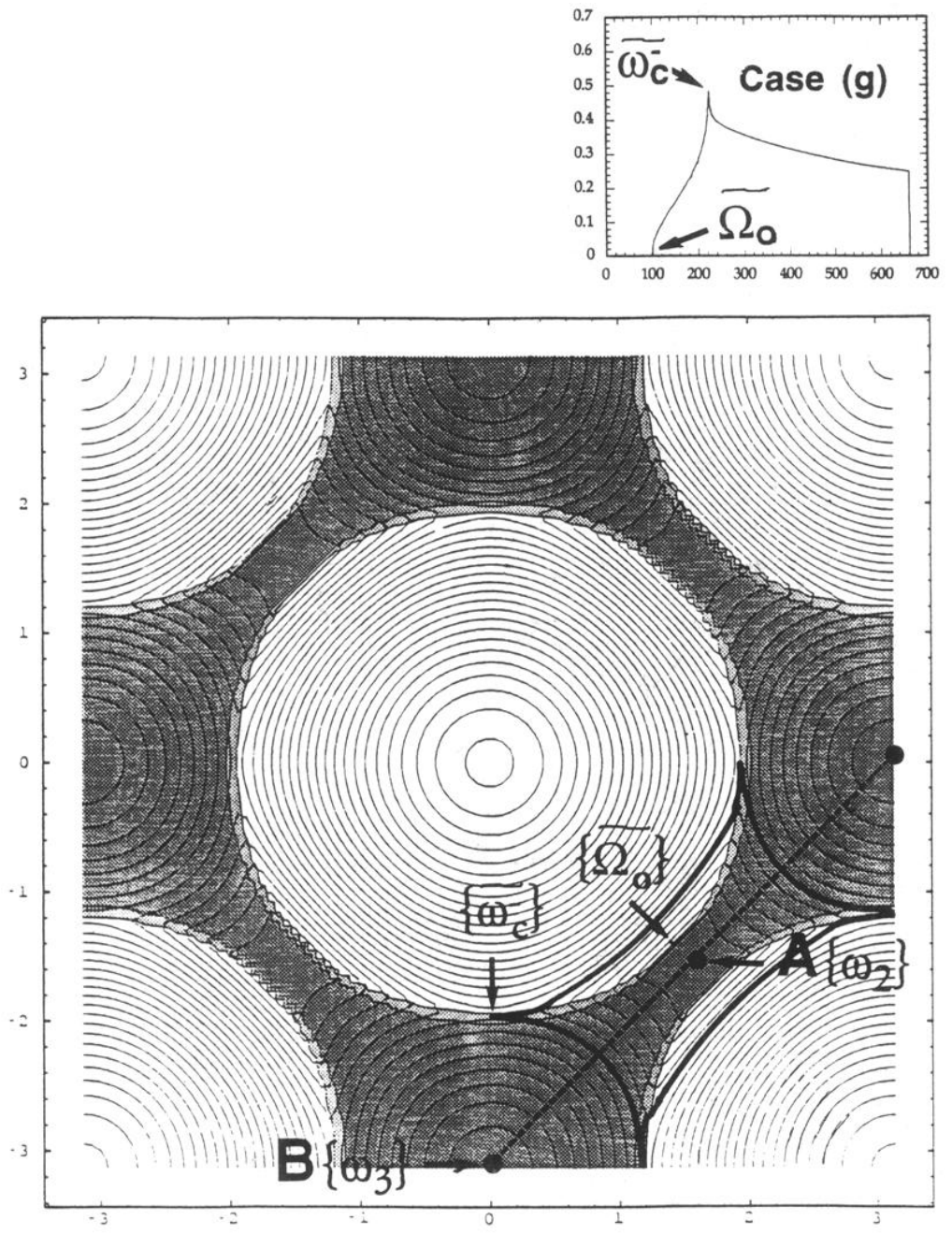

FIG. 11. (Continued).

(d) 

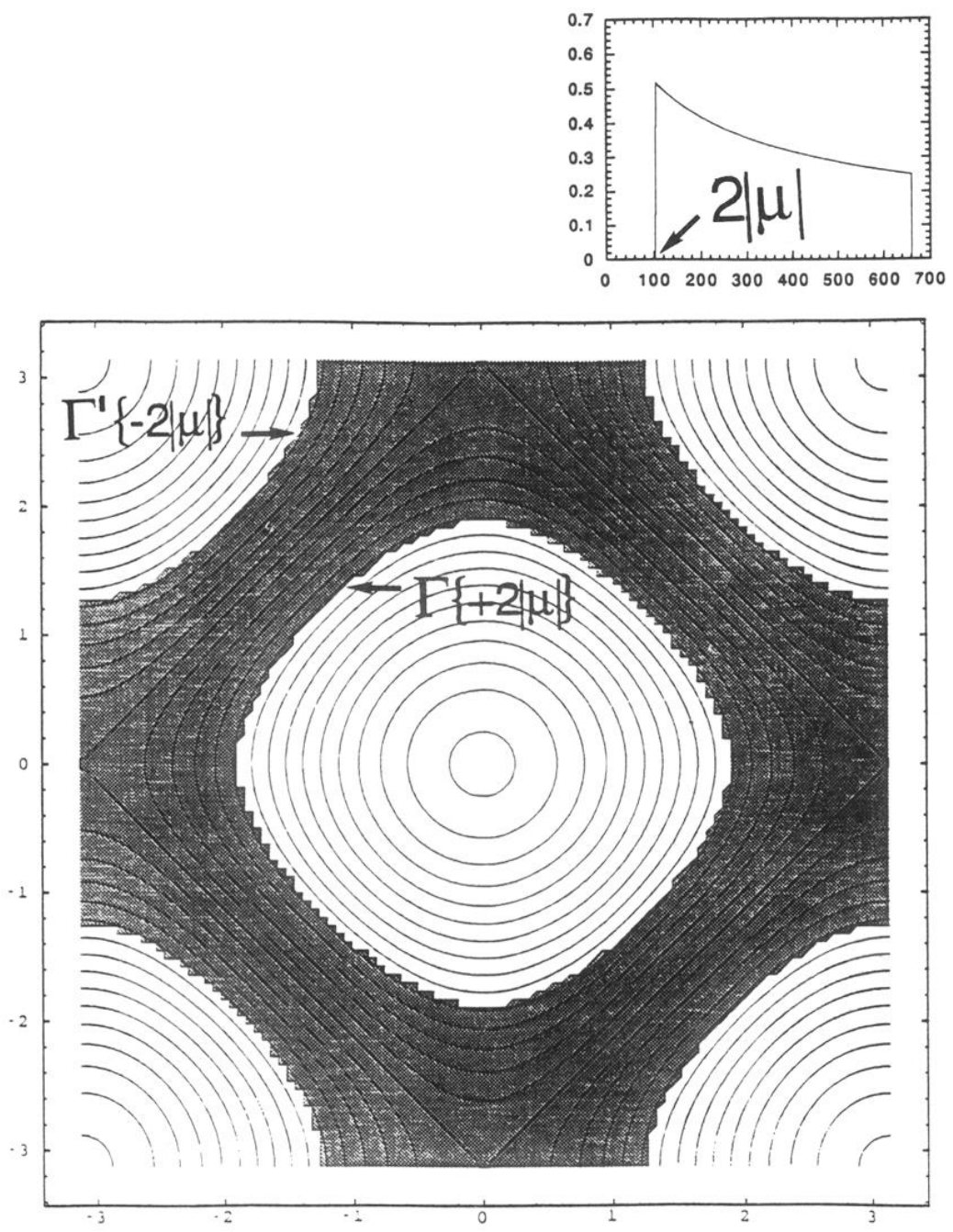

FIG. 6. Equienergetics $\xi_{k+Q}-\xi_{k}=$ const without pairing. The shaded region does not contribute to $\chi_{0}^{\prime \prime}(\mathbf{Q}, \omega)$ because of the extinction role played by the Fermi factor between the curves $\Gamma$ and $\Gamma^{\prime}$. In the inset, spectral weight $\chi_{0}^{\prime \prime}(\mathbf{Q}, \omega)$ vs frequency $\omega$ without pairing at $T=0$ for $t^{\prime}=0$. For $\mu \neq 0$ the vector $\mathbf{Q}$ no longer spans the Fermi surface and a gap of value $2|\mu|$ opens up. 


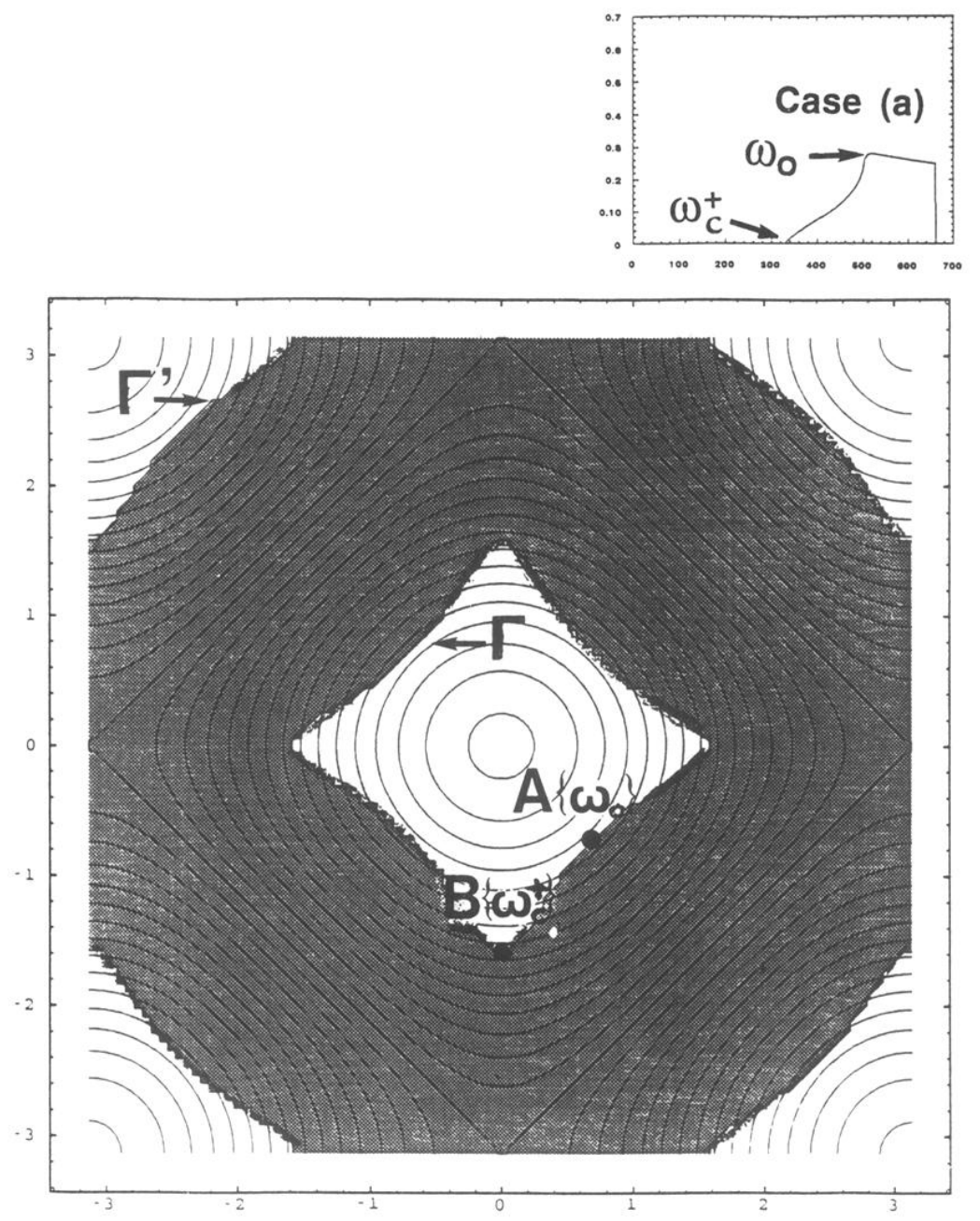

(a)
FIG. 7. Equienergetics $\xi_{k+Q}-\xi_{k}=$ const without pairing at $T=0$ for $t=75 \mathrm{meV}$, $t^{\prime}=-0.45 t$. (a) case (a) $\mu<4 t^{\prime}$ (closed orbits). (b) case (c) $4 t^{\prime}<\mu<\mu_{c 1}$ (large open orbits). (c) case (g) $\mu>0$ (small open orbits). The convention used for the shaded regions is the same as in Fig. 6. In the inset, spectral weight $\chi_{0}^{\prime \prime}(\mathbf{Q}, \omega)$ vs frequency in each case. 

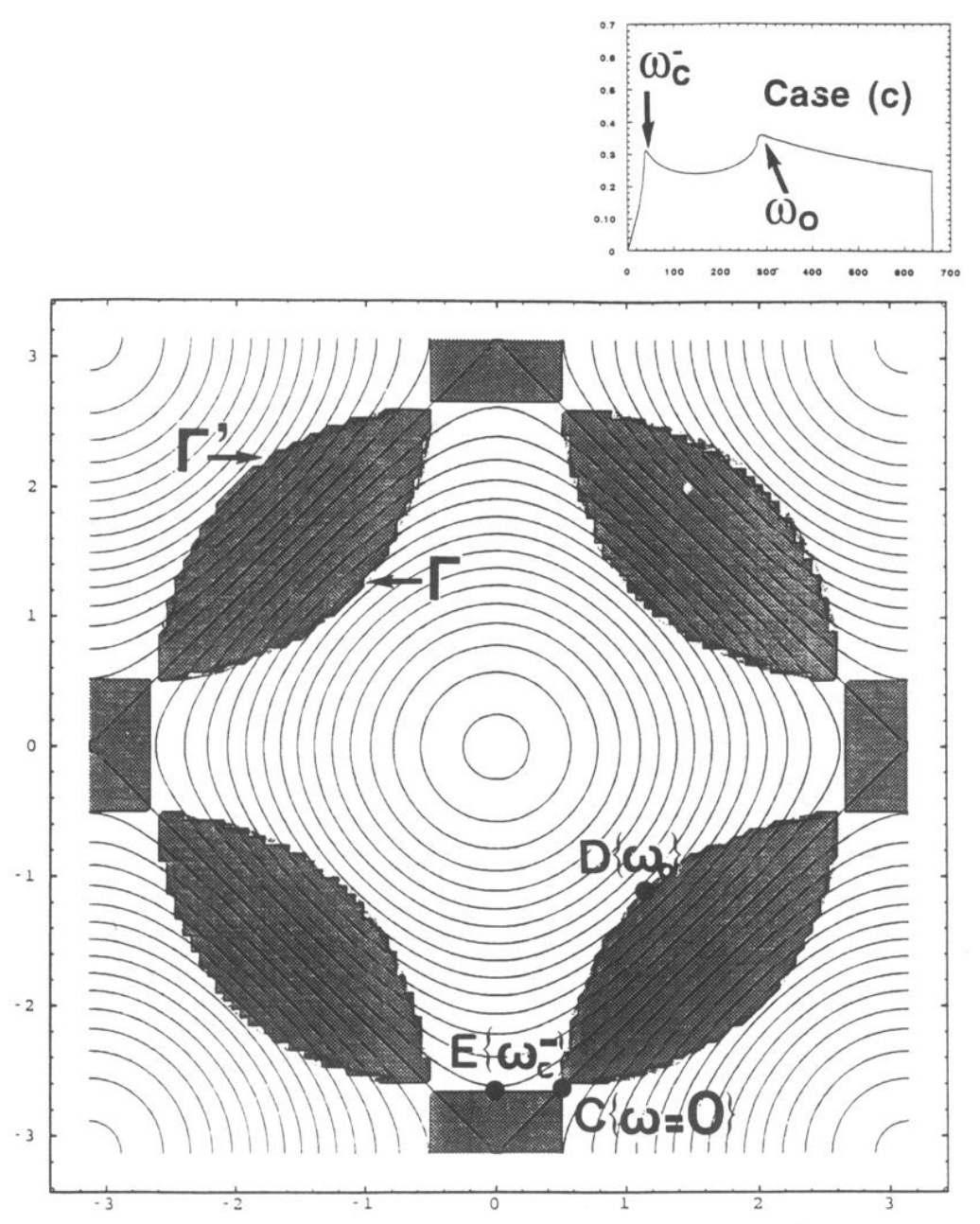

FIG. 7. (Continued).

(b) 

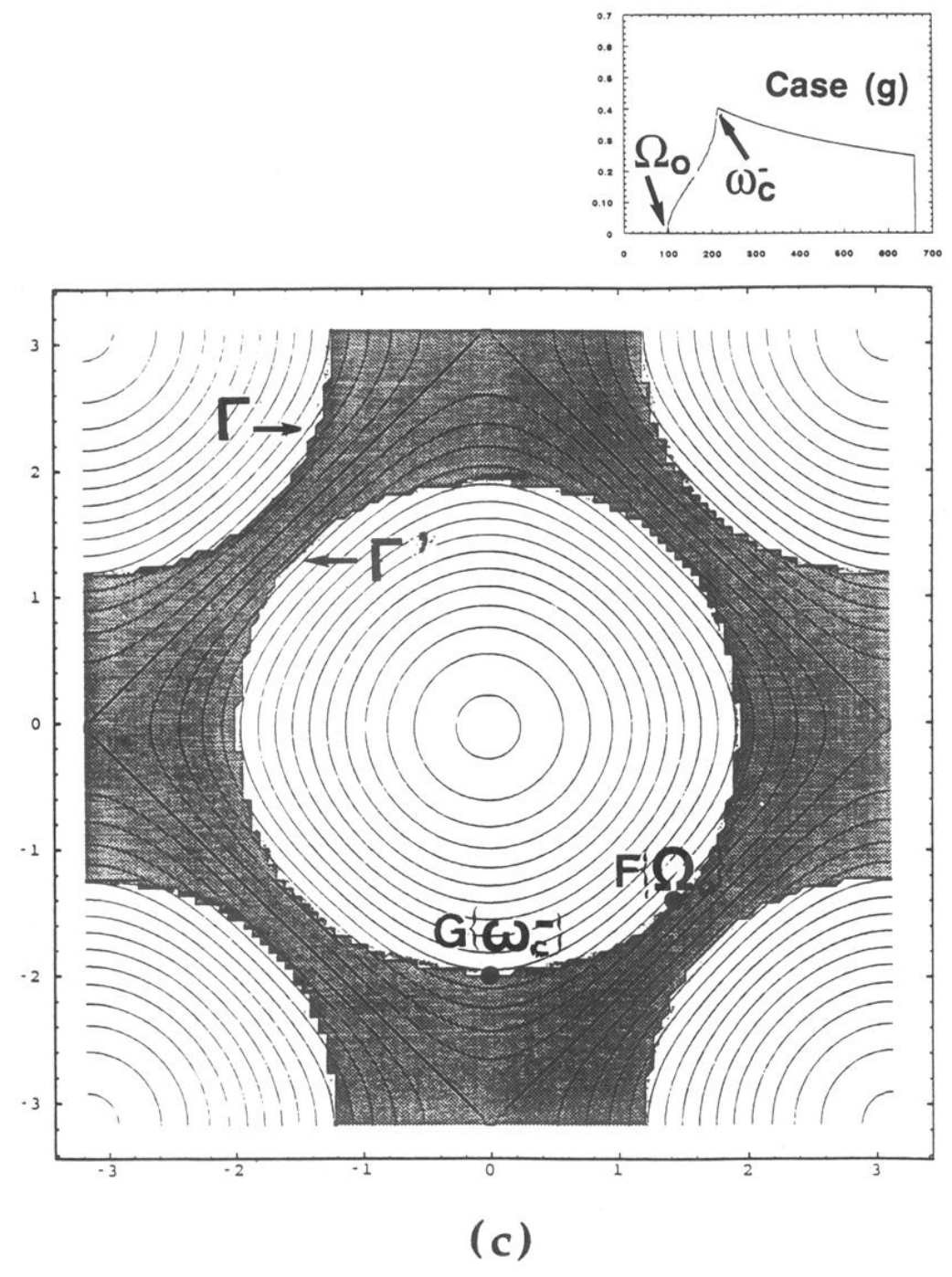

FIG. 7. (Continued). 